\title{
Toward verification of the Riemann hypothesis: Application of the Li criterion
}

\author{
Mark W. Coffey \\ Department of Physics \\ Colorado School of Mines \\ Golden, CO 80401
}

(Received

2004)

August 10, 2004

\begin{abstract}
We substantially apply the Li criterion for the Riemann hypothesis to hold. Based upon a series representation for the sequence $\left\{\lambda_{k}\right\}$, which are certain logarithmic derivatives of the Riemann xi function evaluated at unity, we determine new bounds for relevant Riemann zeta function sums and the sequence itself. We find that the Riemann hypothesis holds if certain conjectured properties of a sequence $\eta_{j}$ are valid. The constants $\eta_{j}$ enter the Laurent expansion of the logarithmic derivative of the zeta function about $s=1$ and appear to have remarkable characteristics. On our conjecture, not only does the Riemann hypothesis follow, but an inequality governing the values $\lambda_{n}$ and inequalities for the sums of reciprocal powers of the nontrivial zeros of the zeta function.
\end{abstract}

\section{Key words and phrases}

Riemann zeta function, Riemann xi function, logarithmic derivatives, Riemann hypothesis, Li criterion, Stieltjes constants, Laurent expansion, L function, Dirichlet series, Hecke L function, Dedekind zeta function, extended Riemann hypothesis 


\section{Introduction}

In this paper, we reduce the verification of the Riemann hypothesis to a conjecture concerning the behavior of certain coefficients $\eta_{k}$ which appear in the Laurent expansion of the logarithmic derivative of the zeta function about $s=1$. Moreover, should the conjectured property hold, we would then derive a result stronger than the Riemann hypothesis itself. Namely, we would have obtained an explicit lower bound for a sequence $\left\{\lambda_{j}\right\}$ of certain logarithmic derivatives of the xi function evaluated at unit argument. Since the coefficients $\eta_{j}$ can be written as particular limits involving the von Mangoldt function $\Lambda$, these quantities seem to encapsulate both number theoretic and analytic information. Such information is expected to be encountered in any rigorous denial or verification of the Riemann hypothesis.

We first present an overview of our approach. We shall use the Li equivalence for the Riemann hypothesis to hold [25]. This equivalence results from a necessary and sufficient condition that the logarithmic derivative of the function $\xi[1 /(1-z)]$ be analytic in the unit disk. The function $\xi$ is determined from the Riemann zeta function $\zeta$ by way of the relation $\xi(s)=(s / 2)(s-1) \pi^{-s / 2} \Gamma(s / 2) \zeta(s)$, where $\Gamma$ is the Gamma function $[13,20,22,38,37]$. Then the xi function satisfies the functional equation $\xi(s)=\xi(1-s)$. The Li equivalence states that a necessary and sufficient condition for the nontrivial zeros of the Riemann zeta function to lie on the critical line $\operatorname{Re} s=1 / 2$ is that a sequence of real numbers $\lambda_{k}$ is nonnegative for every integer $k$. In this paper, we investigate the behavior of this sequence, based upon a series 
representation previously derived [9]. Of this representation, we are able to bound one finite sum and make progress in bounding the other. We find that indeed the sequence $\left\{\lambda_{k}\right\}$ has only nonnegative numbers, subject to the conjectured properties of the sequence $\eta_{j}$. Among other connections, the $\eta_{j}$ can be readily related to the Stieltjes constants $\gamma_{k}$. This gives one of several avenues for further investigation of the $\eta_{j}$ expansion coefficients. This paper includes a series of appendices A-L which contain various extensions of our summation estimations, occasional alternative proofs, series representations analogous to that for $\lambda_{n}$ relevant to other L-functions, tabulated numerical values, derivative relations of the Riemann zeta function, alternative expansions of the logarithmic derivative of the Riemann zeta and xi functions, remarks on the integer-order derivatives of the Dedekind zeta and xi functions, and other reference material.

We stress that we do not just conjecture as to the nature of the $\eta_{j}$ 's, but provide a perhaps strong plausibility argument in favor. In addition, current numerical evidence [29] seems to fully support our conjecture.

The sequence $\left\{\lambda_{n}\right\}_{n=1}^{\infty}$ is defined by

$$
\lambda_{n}=\frac{1}{(n-1) !} \frac{d^{n}}{d s^{n}}\left[s^{n-1} \ln \xi(s)\right]_{s=1} .
$$

Then Li's criterion for the Riemann hypothesis to hold is that all $\left\{\lambda_{n}\right\}_{n=1}^{\infty}$ are nonnegative [25]. We note that Li's convention for the xi function has a factor of two difference: $\xi_{L i}(s)=2 \xi(s)$, although this is immaterial in logarithmic derivatives such 
as $\lambda_{j}$. We also have

$$
\lambda_{n}=\frac{(-1)^{n}}{(n-1) !} \frac{d^{n}}{d s^{n}}\left[(1-s)^{n-1} \ln \xi(s)\right]_{s=0},
$$

and $\xi(0)=-\zeta(0)=1 / 2$. (Hence $\xi_{L i}(0)=1$.) The approximate numerical values for the first few $\lambda_{j}$ 's are: $\lambda_{1} \simeq 0.0230957, \lambda_{2} \simeq 0.0923457$, and $\lambda_{3} \simeq 0.207639$ (see Appendix D). In fact, we have $\lambda_{1}=-B$, where $B \equiv \ln 2+(1 / 2) \ln \pi-1-\gamma / 2 \simeq$ -0.0230957 , and $\gamma \simeq 0.5772156649$ is the Euler constant. This follows from the logarithmic derivative [20]

$$
\frac{\xi^{\prime}(s)}{\xi(s)}=B+\sum_{\rho}\left(\frac{1}{s-\rho}+\frac{1}{\rho}\right),
$$

where $\rho$ runs over all the nontrivial zeros of the zeta function. Thus $\xi^{\prime}(s) / \xi(s)=$ $\sum_{\rho}(s-\rho)^{-1}$, which is consistent with $\xi_{L i}(s)=\prod_{\rho}(1-s / \rho)$. In general, the $\lambda_{j}$ 's are connected to sums over the nontrivial zeros of $\zeta(s)$ by way of [25]

$$
\lambda_{n}=\sum_{\rho}\left[1-\left(1-\frac{1}{\rho}\right)^{n}\right] .
$$

By using the Laurent expansion of the zeta function about $s=1$,

$$
\zeta(s)=\frac{1}{s-1}+\sum_{n=0}^{\infty} \frac{(-1)^{n}}{n !} \gamma_{n}(s-1)^{n},
$$

where the $\gamma_{k}$ are the Stieltjes constants [20,19], it is possible to write a closed form for the $\lambda_{j}$ 's. The Stieltjes constants can be evaluated from the expression

$$
\gamma_{k}=\lim _{N \rightarrow \infty}\left(\sum_{m=1}^{N} \frac{1}{m} \ln ^{k} m-\frac{\ln ^{k+1} N}{k+1}\right),
$$


and several other forms have been given [19]. For instance, we have

$$
\lambda_{2}=1+\gamma-\gamma^{2}+\pi^{2} / 8-2 \ln 2-\ln \pi-2 \gamma_{1}
$$

and

$$
\lambda_{3}=\frac{1}{2}\left[2+\frac{3}{4} \pi^{2}-6 \ln 2-3 \ln \pi-12 \gamma_{1}+\gamma\left[3+2(\gamma-3) \gamma+6 \gamma_{1}\right]+3 \gamma_{2}-\frac{7}{4} \zeta(3)\right]
$$

It is not difficult to determine that $\lambda_{j}$ contains the term $-(-1)^{j}[j /(j-1) !] \gamma_{j-1}$. The successive $\lambda_{j}$ 's can be related in several different ways, including simply

$$
\lambda_{n+1}=\lambda_{n}+\frac{1}{n !}\left[\frac{d^{n}}{d s^{n}} s^{n} \frac{\xi^{\prime}(s)}{\xi(s)}\right]_{s=1}
$$

Alternatively, in the particular case of $\lambda_{2}$, one can write $\lambda_{2}=2 \lambda_{1}-\lambda_{1}^{2}+\lim _{s \rightarrow 1} s \xi^{\prime \prime}(s) / \xi(s)$. Elsewhere, we have very recently obtained a general explicit relation for $\lambda_{k}$ in terms of the Stieltjes constants [11].

\section{Alternative representation of Li's $\lambda_{j}$ 's}

Of particular importance for the purposes of this paper is an alternative formula for the particular sequence of logarithmic derivatives of the Riemann xi function given in Eq. (1). Due to the centrality of this result, we briefly review the derivation [9] of the following representation,

\section{Theorem 1}

$$
\lambda_{n}=-\sum_{m=1}^{n}\left(\begin{array}{l}
n \\
m
\end{array}\right) \eta_{m-1}+\sum_{m=2}^{n}(-1)^{m}\left(\begin{array}{c}
n \\
m
\end{array}\right)\left(1-2^{-m}\right) \zeta(m)+1-\frac{n}{2}(\gamma+\ln \pi+2 \ln 2),
$$


where the constants $\eta_{j}$ can be written as

$$
\eta_{k}=\frac{(-1)^{k}}{k !} \lim _{N \rightarrow \infty}\left(\sum_{m=1}^{N} \frac{1}{m} \Lambda(m) \ln ^{k} m-\frac{\ln ^{k+1} N}{k+1}\right)
$$

and $\Lambda$ is the von Mangoldt function [13, 20, 22, 38, 37, 21]. From the expansion around $s=1$ of the logarithmic derivative of the zeta function,

$$
\frac{\zeta^{\prime}(s)}{\zeta(s)}=-(s-1)^{-1}-\sum_{p=0}^{\infty} \eta_{p}(s-1)^{p}
$$

we have

$$
\ln \zeta(s)=-\ln (s-1)-\sum_{p=1}^{\infty} \frac{\eta_{p-1}}{p}(s-1)^{p}
$$

giving

$$
\ln \xi(s)=-\ln 2+\ln s-\frac{s}{2} \ln \pi+\ln \Gamma(s / 2)-\sum_{p=1}^{\infty} \frac{\eta_{p-1}}{p}(s-1)^{p} .
$$

The radius of convergence of the expansion (12) is 3, as the first singularity encountered is the trivial zero of $\zeta(s)$ at $s=-2$. We then evaluate

$$
\frac{d^{n}}{d s^{n}}\left[s^{n-1} \ln \xi(s)\right]_{s=1}=(n-1) ! \sum_{j=0}^{n-1}\left(\begin{array}{l}
n \\
j
\end{array}\right) \frac{1}{(n-j-1) !}\left[\frac{d^{n-j}}{d s^{n-j}} \ln \xi(s)\right]_{s=1},
$$

using in particular the special values $\psi(1 / 2)=-\gamma-2 \ln 2$ and $\psi^{(n)}(1 / 2)=(-1)^{n+1} n !\left(2^{n+1}-\right.$ 1) $\zeta(n+1)$ for $n \geq 1$, where $\psi=\Gamma^{\prime} / \Gamma$ is the digamma function and $\psi^{(j)}$ is the polygamma function. Finally, the sum in Eq. (15) over $j$ can be converted to a sum over $m=n-j$ and the simple result $-\sum_{m=1}^{n}(-1)^{m}\left(\begin{array}{c}n \\ m\end{array}\right)=1$ used, yielding Eq. (10).

The Laurent expansion of $\zeta^{\prime} / \zeta$ with the form of the constants $\eta_{j}$ in Eq. (11) can be developed by applying Theorem 1 of Ref. [21]. In this case, the counting function $A(x)=-\sum_{n \leq x} \Lambda(n)=-\psi(x)$, where $\psi$ is the Chebyshev function, and the error 
term is given by $u(x)=x-\psi(x)$. Equation (10) has been derived alternatively in Ref. [6] by a method connected with A. Weil's explicit formula. Our approach is independent, and we believe, more direct. In Appendix G we present an extension of Theorem 1 which accounts explicitly for the presence of the first six trivial zeros of the zeta function.

\section{Estimation of Sums}

We characterize each of the two summation terms on the right side of Eq. (10) in turn. Before this discussion, we emphasize that the first few $\lambda_{j}$ 's may be explicitly written, as indicated above, and directly verified to be positive. In addition, we note that apparently it is already known [5] that $\lambda_{n} \geq 0$ for all $n \leq 2.975 \ldots \times 10^{17}$.

The sum

$$
S_{1}(n) \equiv \sum_{m=2}^{n}(-1)^{m}\left(\begin{array}{c}
n \\
m
\end{array}\right)\left(1-2^{-m}\right) \zeta(m),
$$

may be written in several equivalent ways, including

$$
S_{1}(n)=\sum_{k=0}^{\infty} \sum_{m=2}^{n}(-1)^{m}\left(\begin{array}{c}
n \\
m
\end{array}\right) \frac{1}{(2 k+1)^{m}}=\sum_{k=0}^{\infty}\left[\frac{n}{2 k+1}-1+\frac{2^{n} k^{n}}{(2 k+1)^{n}}\right] .
$$

Equation (17) results from the use of (e.g., [1])

$$
\left(1-2^{-n}\right) \zeta(n)=\sum_{k=0}^{\infty}(2 k+1)^{-n}, \quad n \geq 2,
$$

and interchange of the order of the two summations in Eq. (16). It appears to be equally profitable to write $S_{1}$ as

$$
S_{1}(n)=\sum_{k=1}^{\infty} \sum_{m=2}^{n}(-1)^{m}\left(\begin{array}{c}
n \\
m
\end{array}\right)\left(1-2^{-m}\right) \frac{1}{k^{m}}=\sum_{k=1}^{\infty}\left[\frac{n}{2 k}+\left(1-\frac{1}{k}\right)^{n}-\left(1-\frac{1}{2 k}\right)^{n}\right] .
$$


By using integrals estimating these forms, we obtain

\section{Theorem 2}

$$
S_{1}(n) \geq \frac{n}{2} \ln n+(\gamma-1) \frac{n}{2}+\frac{1}{2} .
$$

Remark. By inserting an integral representation for $\zeta$ into Eq. (16), it is possible to obtain the sum $S_{1}$ in the form

$$
S_{1}(n)=\frac{n}{2} \int_{0}^{\infty}\left[1+{ }_{1} F_{1}(1-n ; 2 ; t / 2)-2{ }_{1} F_{1}(1-n ; 2 ; t)\right] \frac{d t}{\left(e^{t}-1\right)},
$$

where ${ }_{1} F_{1}$ is the Kummer confluent hypergeometric function. In this equation, $n_{1} F_{1}(1-n ; 2 ; w)=L_{n-1}^{1}(w)$, where $L_{n}^{\alpha}$ is an associated Laguerre polynomial.

Proof of Theorem 2. We proceed on the basis of comparison to the form of Eq. (17), leaving the comparison to Eq. (19) to Appendix A. In addition, we relegate to Appendix L the application of Euler-Maclaurin summation to $S_{1}(n)$, which gives improved estimates of the linear term.

By making a change of variable in the integral

$$
I_{1}(n)=\int_{0}^{\infty}\left[\frac{n}{2 k+1}-1+\frac{2^{n} k^{n}}{(2 k+1)^{n}}\right] d k,
$$

we obtain

$$
I_{1}(n)=\frac{1}{2} \int_{1}^{\infty}\left[\frac{n}{x}-1+\frac{(x-1)^{n}}{x^{n}}\right] d x .
$$

Another change of variable and an integration by parts yields

$$
I_{1}(n)=\frac{n}{2} \int_{0}^{1}\left[1-(1-t)^{n-1}\right] \frac{d t}{t}-\frac{1}{2}(n-1) .
$$


Evaluation of the integration [16] then gives

$$
I_{1}(n)=\frac{n}{2}[\psi(n)+\gamma-1]+\frac{1}{2} .
$$

Since the integrand of Eq. (21) is monotonically decreasing with $k$, the inequality (20) readily follows.

Corollary With the other sum term in Eq. (10) denoted as $S_{2}$, we have already obtained the inequality $\lambda_{n} \geq(n / 2) \ln n-(n / 2)(1+\ln \pi+2 \ln 2)+3 / 2-\left|S_{2}\right|$. Similarly, it is possible to readily obtain an upper bound for $S_{1}$ and $\lambda_{n}$. We have, for instance, $S_{1}(n) \leq(n / 2) \ln n+(\gamma+1) n / 2-1 / 2$. It is possible to develop improved coefficients for the linear in $n$ term, giving tighter bounds for $S_{1}(n)$, as shown in Appendix L.

Remarks. (1) Since Li has very recently obtained explicit formulas for both Dirichlet and Hecke L-functions [26], which are analogous to Eq. (10), we may expect our summation estimation techniques to aid in making progress in verifying the extended and generalized Riemann hypotheses. In fact, we may observe that the term $\tau_{\chi}(n)$ defined in Theorem 1 of Ref. [26] can be rewritten as

$$
\begin{aligned}
& \tau_{\chi}(n)=S_{1}-n \ln 2 \quad \text { if } \quad \chi(-1)=1, \\
& =S_{0}-S_{1}, \quad \text { if } \quad \chi(-1)=-1,
\end{aligned}
$$

where $S_{0}$ is defined in Eq. (A.15) of Appendix A. The second line of Theorem 2 of Ref. [26] for $\lambda_{E}(n)$ may be written as

$$
2\left(1-\frac{1}{3} \ln 2\right) n+S_{3}-S_{0}+2 n+(-1)^{n}-1,
$$


where $S_{3}$ is defined in Eq. (A.18) of Appendix A. Inequality results based upon the use of Eqs. (25) and (26) and the estimations of Appendix A are given in Appendix E, while estimations of generalized Riemann zeta functions sums are presented in Appendix F.

(2) In Ref. [39], the rate of growth of the sum $S_{1}(n)$ is conjectured. An integral expression for the Li constants is written and a saddle point method is applied. However, the dominant $O(n \ln n)$ behaviour that we have demonstrated is not rigorously obtained.

It is not difficult to show that the following recursion relation exists between the Stieltjes constants of Eq. (5) and the coefficients $\eta_{j}$. We have $\eta_{0}=-\gamma_{0}=-\gamma$ and

$$
\eta_{n}=-(-1)^{n}\left[\frac{(n+1)}{n !} \gamma_{n}+\sum_{k=0}^{n-1} \frac{(-1)^{k-1}}{(n-k-1) !} \eta_{k} \gamma_{n-k-1}\right] .
$$

This equation is equivalent to the statement $\left[\zeta^{\prime}(s) / \zeta(s)\right] \zeta(s)=\zeta^{\prime}(s)$ and will be applied later on.

It is also of interest to relate the sequence $\left\{\eta_{j}\right\}$ to the sequence $\left\{\sigma_{k}\right\}$ which occurs in the expansion

$$
\ln \xi(s)=-\ln 2-\sum_{k=1}^{\infty}(-1)^{k} \frac{\sigma_{k}}{k}(s-1)^{k} .
$$

The ensueing relation figures prominently in our conjecture for the behaviour of the coefficients $\eta_{j}$. One reason for the importance of the coefficients $\sigma_{k}$ is their correspondence to sums of reciprocal powers of the nontrivial zeros $\rho$ of the $\zeta$ function $[24,31]$ : $\sigma_{k}=\sum_{\rho} \rho^{-k}$. From Eq. (4) we see that the connection between the values $\lambda_{n}$ and the sequence $\left\{\sigma_{k}\right\}$ is $\lambda_{n}=-\sum_{j=1}^{n}(-1)^{j}\left(\begin{array}{l}n \\ j\end{array}\right) \sigma_{j}$. Further discussion of the $\sigma_{j}$ 's is presented 
in Appendix J. We shall next demonstrate

\section{Theorem 3}

$$
\sigma_{k}=(-1)^{k} \eta_{k-1}-\left(1-2^{-k}\right) \zeta(k)+1, \quad k \geq 2
$$

Proof of Theorem 3. The key to showing that Eq. (29) holds is to evaluate the particular successive Riemann zeta function sums

$$
\sum_{n=k}^{\infty} \frac{[\zeta(n)-1]}{2^{n}}\left(\begin{array}{l}
n-1 \\
k-1
\end{array}\right)=\left(1-2^{-k}\right) \zeta(k)-1, \quad k \geq 2 .
$$

From Eq. (14) and the expansion [1]

$$
\ln \left[s \Gamma\left(\frac{s}{2}\right)\right]=\ln 2+\frac{(\gamma-1)}{2}+\frac{(1-\gamma)}{2}(s-1)+\sum_{n=2}^{\infty} \frac{[\zeta(n)-1]}{n 2^{n}}[1-(s-1)]^{n},
$$

we have

$$
\begin{aligned}
\ln \xi(s)=-\frac{1}{2} \ln \pi & +\frac{\gamma-1}{2}+\sum_{n=2}^{\infty} \frac{[\zeta(n)-1]}{n 2^{n}}+(1-s)\left[\frac{\ln \pi}{2}-\frac{1}{2}(\gamma+1)+\sum_{n=2}^{\infty} \frac{[\zeta(n)-1]}{2^{n}}\right] \\
& -\sum_{p=2}^{\infty} \frac{\eta_{p-1}}{p}(s-1)^{p}+\sum_{n=2}^{\infty} \sum_{j=2}^{n} \frac{[\zeta(n)-1]}{n 2^{n}}\left(\begin{array}{c}
n \\
j
\end{array}\right)(1-s)^{j} .
\end{aligned}
$$

Upon comparison with Eq. (28), the constant term in this equation (i.e., the coefficient of the $(1-s)^{0}$ term) is readily shown to be zero (or see Appendix B) and the linear term immediately gives

$$
\sigma_{1}=-\frac{\ln \pi}{2}+\frac{\gamma}{2}+1-\ln 2=\lambda_{1}
$$

We then have

$$
-\sum_{p=2}^{\infty} \frac{\eta_{p-1}}{p}(s-1)^{p}+\sum_{n=2}^{\infty} \sum_{j=2}^{n} \frac{[\zeta(n)-1]}{n 2^{n}}\left(\begin{array}{c}
n \\
j
\end{array}\right)(1-s)^{j}=-\sum_{k=2}^{\infty} \frac{\sigma_{k}}{k}(1-s)^{k} .
$$


Upon reordering the two sums we obtain

$$
\sigma_{k}=(-1)^{k} \eta_{k-1}-\sum_{n=k}^{\infty} \frac{[\zeta(n)-1]}{2^{n}}\left(\begin{array}{l}
n-1 \\
k-1
\end{array}\right), \quad k \geq 2 .
$$

We now need to demonstrate Eq. (30). We first note that

$$
\sum_{n=k}^{\infty} \frac{[\zeta(n)-1]}{2^{n}}\left(\begin{array}{l}
n-1 \\
k-1
\end{array}\right)=\sum_{\ell=2}^{\infty} \frac{1}{(k-1) !} \sum_{n=k}^{\infty} \frac{(n-1)(n-2) \cdots(n-k+1)}{(2 \ell)^{n}}
$$

and

$$
\sum_{k=j+1}^{\infty} k(k-1)(k-2) \cdots(k-j) q^{k-j-1}=\frac{(j+1) !}{(1-q)^{j+2}}, \quad|q|<1 .
$$

Equation (37) is easily verified by mathematical induction. With Eq. (36), we have proceeded by interchanging two summations. However, we could have equally well used the integral representation

$$
\zeta(s)=\frac{1}{\Gamma(s)} \int_{0}^{\infty} \frac{t^{s-1}}{\left(e^{t}-1\right)} d t, \quad \operatorname{Re} s>1,
$$

and then interchanged the order of summation and integration, which is carried out in Appendix B. By applying Eq. (37) to Eq. (36) we obtain the succinct result

$$
\sum_{n=k}^{\infty} \frac{[\zeta(n)-1]}{2^{n}}\left(\begin{array}{l}
n-1 \\
k-1
\end{array}\right)=\sum_{\ell=1}^{\infty} \frac{1}{(2 \ell+1)^{k}} .
$$

If we then invoke Eq. (18) we obtain successively Eqs. (30) and (29). Theorem 3 is proved in another manner in Appendix C.

Since in Eq. (12) $\zeta^{\prime}(s) / \zeta(s)+(s-1)^{-1}$ is a transcendental function analytic in the disc $|s-1|<3$, there are an infinite number of $\eta_{j}$ 's which are nonzero. From this 
equation we have a good deal of information on sums and moments of this sequence. For instance, we have

$$
\begin{aligned}
& \sum_{p=0}^{\infty} \eta_{p}=-\left[1+\frac{\zeta^{\prime}(2)}{\zeta(2)}\right] \simeq-0.430039<0, \\
& \sum_{p=0}^{\infty}(-1)^{p} \eta_{p}=1-\ln 2 \pi \simeq-0.837877<0,
\end{aligned}
$$

and more generally, by taking successive derivatives,

$$
\sum_{p=j}^{\infty} p(p-1) \cdots(p-j+1) \eta_{p}=-(-1)^{j} j !-\left.\left[\frac{\zeta^{\prime}(s)}{\zeta(s)}\right]^{(j)}\right|_{s=2}
$$

Various formulas for zeta function derivatives, and in particular concerning the logarithmic derivative occurring in this equation, are presented in Appendix H. Furthermore, Eq. (13) gives

$$
\ln \zeta(2)=-\sum_{p=1}^{\infty} \frac{\eta_{p-1}}{p}
$$

and

$$
\ln 2=\sum_{p=1}^{\infty}(-1)^{p} \frac{\eta_{p-1}}{p}
$$

By evaluating Eq. (12) at $s=1 / 2$ and using the functional equation we obtain

$$
\sum_{p=0}^{\infty}\left(-\frac{1}{2}\right)^{p} \eta_{p}=2-\frac{1}{2}\left[\ln \pi-\psi\left(\frac{1}{4}\right)\right]
$$

or

$$
\sum_{p=1}^{\infty}\left(-\frac{1}{2}\right)^{p} \eta_{p}=2-\left[\ln \pi-\gamma+\frac{\pi}{2}+3 \ln 2\right] .
$$

An equation such as (40a) shows that not all the $\eta_{j}$ 's can be positive. In fact, we can argue that there are an infinite number of $\eta_{j}$ 's which are positive, and an infinite 
number which are negative. This result, together with the fact that the sequence $\eta_{j}$ decreases to zero with $j$ increasing to infinity, as shown by either Eq. (40a) or (41), provides a Theorem 4 .

By multiplying Eq. (12) by $s^{q}$ with $q>-1$ and integrating over $s$ from 0 to 1 we obtain

$$
\sum_{p=0}^{\infty}(-1)^{p} B(p+1, q+1) \eta_{p}=-[\psi(q+1)+\gamma]-\int_{0}^{1}\left[s^{q} \frac{\zeta^{\prime}(s)}{\zeta(s)}+\frac{1}{s-1}\right] d s
$$

where $B$ is the Beta function.

We now characterize the behaviour of the sequence $\left\{\eta_{j}\right\}$ as a function of its index. We have

\section{Theorem 5}

$$
\left|\eta_{j-1}\right| \leq\left|\sigma_{j}\right|+2^{-j} \zeta(j)
$$

and

Conjecture 1 (i) The sequence $\left\{\sigma_{j}\right\}$ decays with $j$ faster than $1 / 3^{j}$. Then, (ii) for $j$ sufficiently large, the sequence $\left\{\eta_{j}\right\}$ alternates in sign and decreases in magnitude as approximately $1 / 3^{j}$. (iii) For all $j \geq 0$, the magnitudes $\left|\eta_{k}\right|$ satisfy $\left|\eta_{k}\right| \leq \gamma 2^{-k}$. Furthermore, (iv) the sequence $\left\{\eta_{j}\right\}$ alternates in sign for all $j \geq 0$.

Remark. Since the radius of convergence of the expansion (12) is 3 , we know that $\left|\eta_{j}\right|$ cannot increase faster than $1 / 3^{j}$ for sufficiently large $j$. In fact, the expression $\eta_{j} \simeq-\gamma(-1 / 3)^{j}$ for all $j \geq 0$ is a very good approximation. One may readily verify this assertion, for instance, with the sums appearing in Eqs. (40)-(43). In addition, 
all currently known numerical evidence [29] supports both this approximation and the strict sign alternation suggested in the conjecture.

We can not currently prove all parts of Conjecture 1 , but we can offer what we believe to be a strong plausibility argument. In the course of this argument we do prove Theorem 5. In addition, based upon known properties of $\sigma_{j}$, we very recently have proved part (iv) on the strict sign alternation of the $\left\{\eta_{j}\right\}$ sequence for all values of $j[11]$.

(i) The first nontrivial zero $\rho_{1}$ of the zeta function is known to lie on the critical line and to have ordinate approximately given by 14.134725142 (e.g., [33]), i.e., $\rho_{1}=$ $1 / 2+i \alpha_{1}$. This zero, along with its complex conjugate, dominate the sum $\sigma_{k}$ so that for large $k$ we have $\sigma_{k} \sim \alpha_{1}^{-k}$, a rate of decrease much faster than $3^{-k}$. In fact, depending upon whether $k$ is even or odd, and if even, divisible or not by 4 , a leading behaviour of $\sigma_{k}$ is given by one of the four forms $\pm 2 \alpha_{1}^{k} /\left(1 / 4+\alpha_{1}^{2}\right)^{k}$ (for $k$ even) or $\pm k \alpha_{1}^{k-1} /\left(1 / 4+\alpha_{1}^{2}\right)^{k}$ (for $k$ odd). In addition, one may argue much more conservatively with the expression

$$
\begin{array}{cc}
\left(\frac{1}{2}-i \alpha_{1}\right)^{k}+\left(\frac{1}{2}+i \alpha_{1}\right)^{k}= & 2 \sum_{j=0}^{k}\left(\begin{array}{c}
k \\
j
\end{array}\right) \frac{\alpha_{1}^{j}}{2^{k-j}} \times \\
1 & j=4 m \\
0 & j=4 m+1 \\
-1 & j=4 m+2 \\
0 & j=4 m+3
\end{array}
$$

by taking the approximate largest value of the binomial coefficient and ignoring the attenuating effect of the powers of $1 / 2$. This will yield a form of approximately $\sigma_{k} \sim(2 / \sqrt{k})\left(2 / \alpha_{1}\right)^{k}$, which is still a much faster decrease with $k$ than $1 / 3^{k}$. 
For both emphasis and clarity, we restate Eq. (29) as

$$
\eta_{j-1}=(-1)^{j}\left[\sigma_{j}+\left(1-2^{-j}\right) \zeta(j)-1\right], \quad j \geq 2
$$

By appeal to Eq. (18) we have

$$
2^{-j} \zeta(j)>\left(1-2^{-j}\right) \zeta(j)-1=\sum_{k=1}^{\infty} \frac{1}{(2 k+1)^{j}}>\frac{1}{3^{j}}, \quad j \geq 2
$$

Applying the triangle inequality to Eq. (47) and using the left inequality in (48) gives Theorem 5.

Continuing with the argument for part (ii) of conjecture 1, by part (i), (1 $\left.2^{-j}\right) \zeta(j)-1>0$ then dominates in the brackets in Eq. (47) and the sign alternation of the sequence $\left\{\eta_{j}\right\}$ then follows for sufficiently large $j$. (iii) With the aid of the recursion relation (27) or by several other means, it is possible to calculate $\eta_{k}$ for any desired initial set $k=1, \ldots, k_{0}$ and directly verify their sign alternation and the stated inequality (e.g., as in Appendix D). For larger values of $k$, this inequality may hold due to the left inequality in (48) when combined with Eq. (47).

Remark. It is not essential to our main purpose here, but we may comment on the sign pattern of the $\left\{\sigma_{k}\right\}$ sequence. The initial, and in a sense typical, sign pattern is simply $--++--\ldots$, with $\sigma_{1}>0$. Initially, as $k$ takes on the respective values $4 m, 4 m+1,4 m+2,4 m+3$, where $m$ is a positive integer, the sign of $\sigma_{k}$ is given by ,,,++-- . This pattern continues to the point where $k(k-1)>8 \alpha_{1}^{2}$. This explains why $\sigma_{46}>0$ rather than $\sigma_{46}<0$. Similar considerations apply for larger values of $k$ in this sequence. 
Mainly for reference purposes, we will now indicate other possible uses of the recursion relation (27). It has been proved that [4]

$$
\left|\gamma_{n}\right| \leq \frac{\left[3+(-1)^{n}\right](n-1) !}{\pi^{n}}, \quad n \geq 1
$$

which has been improved to [31]

$$
\left|\gamma_{n}\right| \leq \frac{\left[3+(-1)^{n}\right](2 n) !}{n^{n+1}(2 \pi)^{n}}, \quad n \geq 1
$$

As an illustration of the use of such results, the combination of Eqs. (27) and (49) gives

\section{Lemma 1}

$$
\left|\eta_{n}\right| \leq \frac{1}{\pi^{n}}\left\{\frac{(n+1)}{n}\left[3+(-1)^{n}\right]+\sum_{k=1}^{n-1} \frac{\left[3+(-1)^{n-k}\right] \pi^{k+1}}{(n-k)}\left|\eta_{k-1}\right|\right\}+\gamma^{2}, \quad n \geq 1
$$

Similarly, the inequality (50) may be applied to Eq. (27), permitting, for example, inductive arguments on $\left|\eta_{n}\right|$, but we have already deduced Theorem 5 .

We are now in position to estimate the sum

$$
S_{2}(n) \equiv-\sum_{m=1}^{n}\left(\begin{array}{l}
n \\
m
\end{array}\right) \eta_{m-1}
$$

of Eq. (10). On conjecture 1, we have that the $\eta_{j}$ 's decrease in magnitude with $j$ and always alternate in sign. This means that, similar to the behaviour of the sum $S_{1}$, there is a near exponential amount of cancellation in the sum $S_{2}$. Indeed, we have

\section{Conjecture 2}

$$
\left|S_{2}\right| \leq 3 \gamma+C_{2} n^{1 / 2+\epsilon}
$$


where $C_{2}$ is a positive constant and $\epsilon$ is positive and arbitrarily small. This conjecture is partially motivated by our discussion elsewhere [11] of the possible connection between the Stieltjes and Li constants and Brownian motion [5].

The combination of Eq. (10) and the inequalities (20) and (53) results in

\section{Conjecture 3}

$$
\lambda_{n} \geq \frac{n}{2} \ln n-\frac{n}{2}(1+\ln \pi+2 \ln 2)+\frac{3}{2}-3 \gamma-C_{2} n^{1 / 2+\epsilon},
$$

where the approximate numerical value of the coefficient $1+\ln \pi+2 \ln 2$ is 3.53 . This estimation would show, in the absence of the last two terms on the right side, that already for values of $n$ exceeding 34 , we would be ensured that all $\lambda_{n}$ 's are nonnegative. In addition, as previously mentioned, for smaller values of $n$ one has only to directly calculate these particular logarithmic derivatives, from either Eq. (1), (2), or Eq. (10) itself and verify the nonnegativeness of the $\lambda_{j}$ 's (Appendix D).

\section{Summary and Brief Discussion}

Our program for verification of the celebrated Riemann hypothesis should now be clear. We have invoked the Li equivalence [25], wherein it is necessary to demonstrate the nonnegativity of the sequence $\left\{\lambda_{n}\right\}_{n=1}^{\infty}$. Our starting point has been the reformulation of the definition (1) as the series representation (10), $\lambda_{n}=S_{1}+S_{2}+1-n(\gamma+$ $\ln \pi+2 \ln 2) / 2$. Some attention to the sums $S_{1}$ and $S_{2}$ [Eqs. (16) and (52)] has been required because they exhibit the phenomenon of exponential cancellation. That is,

the binomial coefficient $\left(\begin{array}{l}n \\ m\end{array}\right)$ within the two summands can take on values approaching 
$2^{n} / \sqrt{n}$. The strict sign alternation in the summands of $S_{1}$ and $S_{2}$ is critical. For the sum $S_{1}$ the sign alternation is explicit, while for $S_{2}$ it has to be deduced [11], as in Conjecture 1. It should be mentioned that the line of reasoning suggested here for a complete proof of the Riemann hypothesis does not require strict sign alternation of the sequence $\left\{\eta_{j}\right\}$-it is just that this result could make the estimation of the sum $S_{2}$ much easier. In fact, very recently we have proved the strict sign alternation of this sequence [11]. What is next required is an argument that correlates the magnitude of say $\eta_{j}$ to $\eta_{j+1}$. As previously mentioned, current numerical evidence [29] seems to support Conjecture 1 for the behaviour of the $\left\{\eta_{j}\right\}$ and $\left\{\sigma_{k}\right\}$ sequences. Certainly both further computation and analysis appear to be in order.

In contrast to the Stieltjes constants $\gamma_{j}$, whose sign pattern is not so easy to discern, that of the two sequences $\left\{\eta_{k}\right\}_{k=0}^{\infty}$ and $\left\{\lambda_{k}\right\}_{k=1}^{\infty}$ seems to be remarkably simple. Upon Conjecture 1 and Eqs. (29) and (48), our approach would also yield various inequalities for the sums $\sigma_{k}$ of reciprocal powers of the nontrivial zeros of the zeta function.

To put it very mildly, many implications could follow from the results presented. We may stress that the conjectured behaviour of the sequence $\left\{\eta_{j}\right\}$ has multiple implications for the von Mangoldt and Chebyshev functions, among many others.

We may additionally stress, that should the claims of the propositions and conjectures herein indeed be valid, we would have not only verified the Riemann hypothesis but produced yet a stronger result. Namely, we would have developed the inequality 
(54) for the sequence $\left\{\lambda_{n}\right\}$. Moreover, it seems that an asymptotic version of inequality (54) will suffice to verify the Riemann hypothesis since evidently [5] so many $\lambda_{j}$ 's are already known to be nonnegative.

The approach of this paper suggests that verification of the Riemann hypothesis may be possible within analysis. Indeed, our approach may be amenable to confronting the generalized Riemann hypothesis. Some of our sum estimations carry over immediately to the explicit formulas for $\lambda_{\chi}(n)$ and $\lambda_{E}(n)$ in Theorems 1 and 2 respectively in Ref. [26]. In turn, one is left with estimating sums which contain the von Mangoldt function, reciprocal powers of $k$, powers of $\ln k$, and Dirichlet characters. To us, this appears to be a realistic approach to the generalized Riemann hypothesis for Dirichlet and Hecke L-functions and the Dedekind zeta function. We include in Appendix E example results along this line of investigation.

\section{Acknowledgement}

I thank J. C. Lagarias for useful discussion concerning a fall 2003 version of this manuscript. 


\section{Figure Caption}

FIG. 1. Diagram of consecutively increasing circles of convergence corresponding to the inclusion of the first six trivial zeros of the Riemann zeta function in the expansion (G.2). The location of the first nontrivial zero $\rho_{1}$ is indicated. 


\section{Appendix A: Estimation of the sum $S_{1}$ of Eq. (16) and of other sums}

Here we present the derivation of the inequality (20) for the sum $S_{1}$ of Eq. (16), based upon the form (19). By making a change of variable in the integral

$$
I_{1}(n) \equiv \int_{1}^{\infty}\left[\frac{n}{2 k}+\left(1-\frac{1}{k}\right)^{n}-\left(1-\frac{1}{2 k}\right)^{n}\right] d k
$$

we obtain

$$
I_{1}(n)=\int_{0}^{1}\left[\frac{n}{2} y+(1-y)^{n}-\left(1-\frac{y}{2}\right)^{n}\right] \frac{d y}{y^{2}} .
$$

We then evaluate this integral with an integration by parts, resulting in

$$
I_{1}(n)=\frac{n}{2}\left\{\psi(n)+\gamma-1+\int_{0}^{1}\left[\left(1-\frac{y}{2}\right)^{n-1}-(1-y)^{n-1}\right] \frac{d y}{y}\right\}+2^{-n}
$$

where $\psi$ is the digamma function. Since the remaining integral is nonsingular and $\mathrm{O}(1)$ the inequality (20) follows. However, we may continue much further.

By adding and subtracting 1 in the integrand in Eq. (A.3), we may write

$$
I_{1}(n)=\frac{n}{2}[\psi(n)+\gamma-1]+\frac{n}{2} \int_{0}^{1}\left[\left(1-\frac{y}{2}\right)^{n-1}-1\right] \frac{d y}{y}+\frac{n}{2}[\psi(n)+\gamma]+2^{-n}
$$

where

$$
\int_{0}^{1}\left[\left(1-\frac{y}{2}\right)^{n-1}-1\right] \frac{d y}{y}=-\psi(n)-\gamma-\int_{1 / 2}^{1} \frac{(1-w)^{n-1}}{w} d w+\ln 2 .
$$

The integral on the right side of Eq. (A.5) may be evaluated in multiple ways. A first method is to use the Gauss hypergeometric function ${ }_{2} F_{1}$ :

$$
\int_{1 / 2}^{1} \frac{(1-w)^{n-1}}{w} d w=\frac{1}{2^{n-1}} \int_{0}^{1} \frac{(1-x)^{n-1}}{1+x} d x=\frac{1}{n 2^{n-1}}{ }_{2} F_{1}(1,1 ; n+1 ;-1),
$$


where

$$
{ }_{2} F_{1}(1,1 ; n+1 ;-1)=\sum_{j=0}^{\infty}(-1)^{j} \frac{j !}{(n+1)_{j}}=n ! \sum_{j=0}^{\infty}(-1)^{j} \frac{j !}{(j+n) !},
$$

and $(.)_{k}$ is the Pochhammer symbol. By using a partial fractional decomposition of the summand in Eq. (A.7), it is possible to show that the sum contains a term $n 2^{n-1} \ln 2$, leading to a possibly new reduction of the particular ${ }_{2} F_{1}$ at minus unit argument. By applying the well known formula (e.g., [17])

$$
\frac{N !}{x(x+1) \cdots(x+N)}=\sum_{k=0}^{N}\left(\begin{array}{c}
N \\
k
\end{array}\right) \frac{(-1)^{k}}{x+k},
$$

whose right side is simply $(-1)^{N} \Delta^{N}(1 / x)$, where $\Delta$ is the difference operator, $\Delta f(x)=$ $f(x+1)-f(x)$, we obtain

$$
\begin{gathered}
{ }_{2} F_{1}(1,1 ; n+1 ;-1)=n \sum_{k=0}^{n-1}(-1)^{k}\left(\begin{array}{c}
n-1 \\
k
\end{array}\right) \sum_{j=0}^{\infty} \frac{(-1)^{j}}{(j+k+1)} \\
=n\left[2^{n-1} \ln 2-\sum_{k=0}^{n-1}\left(\begin{array}{c}
n-1 \\
k
\end{array}\right) \sum_{j=0}^{k-1} \frac{(-1)^{j}}{j+1}\right] .
\end{gathered}
$$

Next, we proceed alternatively. By way of binomial expansion in the integrand of the second integral in Eq. (A.6), we have

$$
\int_{0}^{1} \frac{(1-x)^{n-1}}{1+x} d x=\sum_{j=0}^{n-1}(-1)^{j}\left(\begin{array}{c}
n-1 \\
j
\end{array}\right) \beta(j+1),
$$

where $[16]$

$$
\beta(j+1)=(-1)^{j} \ln 2+\sum_{k=1}^{j} \frac{(-1)^{k+j}}{k},
$$

giving

$$
\int_{1 / 2}^{1} \frac{(1-w)^{n-1}}{w} d w=\ln 2+\frac{1}{2^{n-1}} \sum_{j=0}^{n-1}\left(\begin{array}{c}
n-1 \\
j
\end{array}\right) \sum_{k=1}^{j} \frac{(-1)^{k}}{k} .
$$


This result is in agreement with Eqs. (A.6) and (A.9). The sum in Eq. (A.12) is easily estimated, leading to

$$
-1+\frac{1}{2^{n}} \leq \frac{n}{2} \int_{1 / 2}^{1} \frac{(1-w)^{n-1}}{w} d w \leq 1-\frac{1}{2^{n}} .
$$

Using Eqs. (A.4), (A.5), (A.12), and (A.13) gives

$$
I_{1}(n) \geq \frac{n}{2}[\psi(n)+\gamma+\ln 2-1]-1+2^{1-n} .
$$

The approach followed here can be used to estimate many other Riemann zeta function sums of interest. For instance, we have

$$
S_{0}(n) \equiv \sum_{m=2}^{n}(-1)^{m}\left(\begin{array}{c}
n \\
m
\end{array}\right) \zeta(m)=\sum_{k=1}^{\infty}\left[\frac{n}{k}-1+\left(1-\frac{1}{k}\right)^{n}\right] .
$$

Then one can determine

$$
I_{0}(n)=\int_{1}^{\infty}\left[\frac{n}{k}-1+\left(1-\frac{1}{k}\right)^{n}\right] d k=\sum_{j=2}^{n}\left(\begin{array}{c}
n \\
j
\end{array}\right) \frac{(-1)^{j}}{(j-1)}
$$

obtained by binomial expansion and term-by-term integration. On the other hand, by using

$$
I_{0}(n)=\int_{0}^{1}\left[n y-1+(1-y)^{n}\right] \frac{d y}{y^{2}},
$$

and an integration by parts, we have $S_{0} \geq I_{0}=n[\psi(n)+\gamma-1]+1$. Since the digamma function satisfies [1] $\psi(x)=\ln x-1 / 2 x-1 / 12 x^{2}+O\left(x^{-4}\right)$ as $x \rightarrow \infty$, the inequality $S_{0}(n) \geq n(\ln n+\gamma-1)+1$ follows for $n \geq 2$.

Additionally, we may estimate

$$
S_{3}(n) \equiv \sum_{j=2}^{n}(-1)^{j}\left(\begin{array}{l}
n \\
j
\end{array}\right) 2^{j} \zeta(j) .
$$


Then we have the comparison integral

$$
I_{3}(n)=\int_{1}^{\infty}\left[\frac{2 n}{k}-1+\left(1-\frac{2}{k}\right)^{n}\right] d k=\int_{0}^{1}\left[2 n y-1+(1-2 y)^{n}\right] \frac{d y}{y^{2}} .
$$

An integration by parts gives

$$
I_{3}(n)=2 n \int_{0}^{1}\left[1-(1-2 y)^{n-1}\right] \frac{d y}{y}-2 n+1-(-1)^{n}
$$

and then we have

$$
I_{3}=(-1)^{n} n\left\{\psi\left(\frac{1-n}{2}\right)-\psi\left(\frac{n}{2}\right)+2(-1)^{n}[\gamma+\ln 2+\psi(n)]+\pi \tan \left(\frac{n \pi}{2}\right)\right\}-2 n+1-(-1)^{n}
$$

One may note that the last three terms on the right side of Eq. (A.21) enter with opposite signs from Eq. (26). The form of $I_{3}$ in Eq. (A.21) may be further rewritten with the use of the relation $[16] \psi[(1-n) / 2]=\psi[(n+1) / 2]-\pi \tan (n \pi / 2)$, together with the doubling formula for the digamma function, $\psi[(n+1) / 2]=2 \psi(n)-\psi(n / 2)-2 \ln 2$. The result is

$I_{3}(n)=2 n\left\{\left[1+(-1)^{n}\right] \psi(n)-(-1)^{n} \psi\left(\frac{n}{2}\right)+\gamma+\left[1-(-1)^{n}\right] \ln 2\right\}-2 n+1-(-1)^{n}$,

which we find to be very useful in Appendix E.

Since we have developed many estimates based upon the digamma function, it may be useful to record another inequality for this function. By way of Binet's first formula [16]

$$
\ln \Gamma(z)=(z-1 / 2) \ln z-z+\frac{1}{2} \ln 2 \pi+\int_{0}^{\infty}\left(\frac{1}{2}-\frac{1}{t}+\frac{1}{e^{t}-1}\right) \frac{e^{-t z}}{t} d t
$$


we have

$$
\psi(z)-\ln z=-\frac{1}{z}+\int_{0}^{\infty}\left(\frac{1}{t}-\frac{1}{e^{t}-1}\right) e^{-t z} d t
$$

By performing manipulations on this representation, it is then possible to show that

$$
-\frac{1}{2 z}-\frac{1}{12 z^{2}}<\psi(z)-\ln z<-\frac{1}{2 z}
$$

The right inequality in (A.25) also follows immediately from the fact $1 / t-1 /\left(e^{t}-1\right)<$ $1 / 2$ proved in Ref. [8].

Another way to proceed in estimating a finite alternating sum such as $S_{1}$ is to rewrite it as a contour integral $[23,15]$,

$$
\sum_{k=\ell}^{N}\left(\begin{array}{l}
N \\
k
\end{array}\right)(-1)^{k} f(k)=-\frac{1}{2 \pi i} \int_{C} B(N+1,-z) f(z) d z
$$

where $C$ is a positively oriented closed curve surrounding the points $\ell, \ell+1, \ldots, N$, $B(x, y)=\Gamma(x) \Gamma(y) / \Gamma(x+y)$ is the Beta function, and $f(z)$ is an analytic continuation of the discrete sequence $f(k)$ to the complex plane, with no poles within the region surrounded by $C$. When the integrand decreases sufficiently rapidly toward $\pm i \infty$, the asymptotic evaluation of this expression can be achieved by extending the contour of the integral to the left and collecting the residues at the newly encountered poles. However, in this paper we have been interested in gaining more information than just an asymptotic evaluation. 


\section{Appendix B: Alternative evaluation of the summation (30)}

Here we perform the sum of Eq. (30) by using the integral representation of Eq. (38) for the zeta function. We also record two simpler sums which are useful in the proof of Theorem 3 .

Upon substituting Eq. (38) into the left side of Eq. (30) we find that

$$
\sum_{n=k}^{\infty} \frac{\zeta(n)}{2^{n}}\left(\begin{array}{l}
n-1 \\
k-1
\end{array}\right)=\frac{1}{2^{k+1}(k-1) !} \int_{0}^{\infty} \frac{t^{k-1} d t}{\sinh (t / 2)}
$$

With a change of variable and evaluation of the integral [16] we have

$$
\sum_{n=k}^{\infty} \frac{\zeta(n)}{2^{n}}\left(\begin{array}{l}
n-1 \\
k-1
\end{array}\right)=\frac{1}{2(k-1) !} \int_{0}^{\infty} \frac{u^{k-1} d u}{\sinh u}=\left(1-2^{-k}\right) \zeta(k)
$$

Then

$$
\sum_{n=k}^{\infty} \frac{[\zeta(n)-1]}{2^{n}}\left(\begin{array}{c}
n-1 \\
k-1
\end{array}\right)=\left(1-2^{-k}\right) \zeta(k)-\frac{1}{2^{k}} \sum_{n=0}^{\infty} \frac{1}{2^{n}}\left(\begin{array}{c}
n+k-1 \\
n
\end{array}\right)=\left(1-2^{-k}\right) \zeta(k)-1
$$

which is Eq. (30).

In connection with the proof of Theorem 3, and Eq. (33) in particular, we write two other zeta function sums:

$$
\begin{gathered}
\sum_{n=2}^{\infty} \frac{[\zeta(n)-1]}{n 2^{n}}=\frac{1-\gamma}{2}+\ln \frac{\sqrt{\pi}}{2} \\
\sum_{n=2}^{\infty} \frac{[\zeta(n)-1]}{2^{n}}=\ln 2-\frac{1}{2}
\end{gathered}
$$




\section{Appendix C: Alternative proof of Theorem 3}

We proceed to deduce Theorem 3 in a way similar to the proof of Theorem 5 of Ref. [31]. In the process, we correct a typographical error which appears in both that proof and Remark 5 and Eq. (1.11) of this reference.

From the definition of the xi function in terms of the zeta function and Eq. (28), we have

$$
\frac{\xi^{\prime}(s)}{\xi(s)}=\frac{1}{s}-\frac{1}{2} \ln \pi+\frac{1}{2} \psi\left(\frac{s}{2}\right)+\frac{\zeta^{\prime}(s)}{\zeta(s)}+\frac{1}{s-1}=\sum_{k=0}^{\infty}(-1)^{k} \sigma_{k+1}(s-1)^{k}
$$

where the sum of the last two terms on the left side is given by Eq. (12) and simply $1 / s=\sum_{j=0}^{\infty}(1-s)^{j}$ for $|1-s|<1$. From the expansion $[16]$

$$
\psi(x)=-\gamma+\sum_{k=2}^{\infty}(-1)^{k} \zeta(k)(x-1)^{k-1}
$$

and the doubling formula satisfied by the digamma function, $\psi(2 z)=\frac{1}{2}[\psi(z)+\psi(z+$ $1 / 2)]+\ln 2$, we obtain

$$
\frac{1}{2} \psi\left(\frac{s}{2}\right)=-\frac{\gamma}{2}+\sum_{k=1}^{\infty}(-1)^{k+1} \zeta(k+1)\left(1-2^{-k-1}\right)(s-1)^{k}-\ln 2 .
$$

The substitution of Eq. (C.3) into Eq. (C.1) and the equating of coefficients of like powers of $s-1$ gives again Eq. (33) for $\sigma_{1}$ from the constant term and Eq. (29) from the rest of the terms. This gives Theorem 3, linking sums of reciprocal powers of the complex zeros of the zeta function with the sequence $\left\{\eta_{j}\right\}$ appearing in the expansion (12) of the logarithmic derivative of the zeta function. 
Appendix D: Tabulated numerical values

\begin{tabular}{|c|c|c|}
\hline$k$ & $\lambda_{k}$ & $\eta_{k}$ \\
\hline 0 & & -0.577216 \\
1 & 0.0230957 & 0.187546 \\
2 & 0.0923457 & -0.0516886 \\
3 & 0.207639 & 0.0147517 \\
4 & 0.368793 & -0.00452448 \\
5 & 0.575543 & 0.0014468 \\
6 & 0.827566 & -0.000471544 \\
7 & 1.12446 & 0.00015518 \\
8 & 1.46576 & -0.0000513452 \\
9 & 1.85092 & 0.0000170414 \\
10 & 2.27934 & $-5.66605 \times 10^{-6}$ \\
11 & 2.75036 & $1.88585 \times 10^{-6}$ \\
12 & 3.26326 & $-6.28055 \times 10^{-7}$ \\
13 & 3.81724 & $2.09241 \times 10^{-7}$ \\
14 & 4.41148 & $-6.97247 \times 10^{-8}$ \\
15 & 5.04508 & $2.32372 \times 10^{-8}$ \\
16 & 5.71711 & $-7.74484 \times 10^{-9}$ \\
17 & 6.42658 & $2.58144 \times 10^{-9}$ \\
18 & 7.17248 & $-8.60444 \times 10^{-10}$ \\
19 & 7.95374 & $2.86808 \times 10^{-10}$ \\
20 & 8.76928 & $-9.56012 \times 10^{-11}$ \\
21 & 9.61796 & $3.18668 \times 10^{-11}$ \\
22 & 10.4986 & $-1.06222 \times 10^{-11}$ \\
23 & 11.4101 & $3.54072 \times 10^{-12}$ \\
24 & 12.3513 & $-1.18024 \times 10^{-12}$ \\
25 & 13.3210 & $3.93412 \times 10^{-13}$ \\
26 & 14.3179 & $-1.31137 \times 10^{-13}$ \\
27 & 15.3408 & $4.37124 \times 10^{-14}$ \\
28 & 16.3885 & $-1.45708 \times 10^{-14}$ \\
29 & 17.4599 & $4.85694 \times 10^{-15}$ \\
30 & 18.5538 & $-1.61898 \times 10^{-15}$ \\
\hline
\end{tabular}




\begin{tabular}{|c|c|c|}
\hline$k$ & $\lambda_{k}$ & $\eta_{k}$ \\
\hline 31 & 19.6689 & $5.3966 \times 10^{-16}$ \\
32 & 20.8041 & $-1.79887 \times 10^{-16}$ \\
33 & 21.9582 & $5.99622 \times 10^{-17}$ \\
34 & 23.1301 & $-1.99874 \times 10^{-17}$ \\
35 & 24.3188 & $6.66247 \times 10^{-18}$ \\
36 & 25.5232 & $-2.22082 \times 10^{-18}$ \\
37 & 26.7422 & $7.40274 \times 10^{-19}$ \\
38 & 27.9749 & $-2.46755 \times 10^{-19}$ \\
39 & 29.2202 & $8.22527 \times 10^{-20}$ \\
40 & 30.4774 & $-2.74176 \times 10^{-20}$ \\
41 & 31.7454 & $9.13919 \times 10^{-21}$ \\
42 & 33.0236 & $-3.0464 \times 10^{-21}$ \\
43 & 34.3111 & $1.01547 \times 10^{-21}$ \\
44 & 35.6072 & $-3.38488 \times 10^{-22}$ \\
45 & 36.9113 & $1.12829 \times 10^{-22}$ \\
46 & 38.2227 & $-3.76098 \times 10^{-23}$ \\
47 & 39.5408 & $1.25366 \times 10^{-23}$ \\
48 & 40.8653 & $-4.17887 \times 10^{-24}$ \\
49 & 42.1955 & $1.39295 \times 10^{-24}$ \\
50 & 43.5311 & $-4.64318 \times 10^{-25}$ \\
51 & 44.8718 & $1.54773 \times 10^{-25}$ \\
52 & 46.2172 & $-5.15909 \times 10^{-26}$ \\
53 & 47.5671 & $1.71970 \times 10^{-26}$ \\
54 & 48.9214 & $-5.73232 \times 10^{-27}$ \\
55 & 50.2798 & $1.91077 \times 10^{-27}$ \\
56 & 51.6423 & $-6.36925 \times 10^{-28}$ \\
57 & 53.0089 & $2.12308 \times 10^{-28}$ \\
58 & 54.3795 & $-7.07695 \times 10^{-29}$ \\
59 & 55.7542 & $2.35898 \times 10^{-29}$ \\
60 & 57.1331 & $-7.86327 \times 10^{-30}$ \\
\hline
\end{tabular}




\begin{tabular}{|c|c|c|}
\hline$k$ & $\lambda_{k}$ & $\eta_{k}$ \\
\hline 61 & 58.5163 & $2.62109 \times 10^{-30}$ \\
62 & 59.9039 & $-8.73697 \times 10^{-31}$ \\
63 & 61.2962 & $2.91232 \times 10^{-31}$ \\
64 & 62.6934 & $-9.70775 \times 10^{-32}$ \\
65 & 64.0957 & $3.23591 \times 10^{-32}$ \\
66 & 65.5033 & $-1.07864 \times 10^{-32}$ \\
67 & 66.9167 & $3.59546 \times 10^{-33}$ \\
68 & 68.3361 & $-1.19849 \times 10^{-33}$ \\
69 & 69.7618 & $3.99496 \times 10^{-34}$ \\
70 & 71.1942 & $-1.33165 \times 10^{-34}$ \\
71 & 72.6337 & $4.43884 \times 10^{-35}$ \\
72 & 74.0805 & $-1.47961 \times 10^{-35}$ \\
73 & 75.5350 & $4.93205 \times 10^{-36}$ \\
74 & 76.9976 & $-1.64402 \times 10^{-36}$ \\
75 & 78.4686 & $5.48005 \times 10^{-37}$ \\
76 & 79.9484 & $-1.82668 \times 10^{-37}$ \\
77 & 81.4373 & $6.08895 \times 10^{-38}$ \\
78 & 82.9357 & $-2.02964 \times 10^{-38}$ \\
79 & 84.4437 & $6.76550 \times 10^{-39}$ \\
80 & 85.9617 & $-2.25516 \times 10^{-39}$ \\
81 & 87.4900 & $7.51722 \times 10^{-40}$ \\
82 & 89.0288 & $-2.50574 \times 10^{-40}$ \\
83 & 90.5782 & $8.35246 \times 10^{-41}$ \\
84 & 92.1386 & $-2.78415 \times 10^{-41}$ \\
85 & 93.7099 & $9.28051 \times 10^{-42}$ \\
86 & 95.2924 & $-3.09350 \times 10^{-42}$ \\
87 & 96.8862 & $1.03117 \times 10^{-42}$ \\
88 & 98.4912 & $-3.43723 \times 10^{-43}$ \\
89 & 100.1076 & $1.14574 \times 10^{-43}$ \\
90 & 101.7352 & $-3.81914 \times 10^{-44}$ \\
\hline
\end{tabular}




\begin{tabular}{|c|c|c|}
\hline$k$ & $\lambda_{k}$ & $\eta_{k}$ \\
\hline 91 & 103.3741 & $1.27304 \times 10^{-44}$ \\
92 & 105.0242 & $-4.24349 \times 10^{-45}$ \\
93 & 106.6852 & $1.41450 \times 10^{-45}$ \\
94 & 108.3572 & $-4.71500 \times 10^{-46}$ \\
95 & 110.0398 & $1.57166 \times 10^{-46}$ \\
96 & 111.7328 & $-5.23888 \times 10^{-47}$ \\
97 & 113.4361 & $1.74629 \times 10^{-47}$ \\
98 & 115.1492 & $-5.82098 \times 10^{-48}$ \\
99 & 116.8719 & $1.94032 \times 10^{-48}$ \\
100 & 118.6038 & $-6.46775 \times 10^{-49}$ \\
\hline
\end{tabular}




\section{Appendix EI: Sum estimations and lower bounds pertinent to other Dirichlet functions}

Analogous to the Corollary which we have presented below Eq. (24) of the text, here we develop similar lower bounds appropriate for explicit formulas for Dirichlet and Hecke L-functions. We make substantial use of the very recent results of Ref. [26], of which we need to recall some details. We relegate to the end of this first part of the Appendix some relations concerning elementary sums. In the second part, we provide independent derivations of the major results, Theorems 1 and 2, of Ref. [26].

Let $\chi$ be a primitive Dirichlet character of modulus $r$, and $L(s, \chi)$ the Dirichlet L-function of character $\chi$. The function

$$
\xi(s, \chi)=\left(\frac{\pi}{r}\right)^{-(s+a) / 2} \Gamma\left(\frac{s+a}{2}\right) L(s, \chi)
$$

where $a$ is 0 if $\chi(-1)=1$ and $a$ is 1 if $\chi(-1)=-1$, satisfies the functional equation $\xi(s, \chi)=\epsilon_{\chi} \xi(1-s, \bar{\chi})$, with $\epsilon_{\chi}$ a constant of absolute value one. The function $\xi(s, \chi)$ is an entire function of order one and has a product representation $\xi(s, \chi)=$ $\xi(0, \chi) \prod_{\rho}(1-s / \rho)$, where the product is over all the zeros of $\xi(s, \chi)$.

We put

$$
\lambda_{\chi}(n)=\sum_{\rho}\left[1-\left(1-\frac{1}{\rho}\right)^{n}\right], \quad n \geq 1 .
$$

We presume that $\lambda_{\chi}(n)>0$ for all $n=1,2, \ldots$ if and only if all of the zeros of $\xi(s, \chi)$ are located on the critical line $\operatorname{Re} s=1 / 2$. Then, Li has obtained [26]

$$
\lambda_{\chi}(n)=S_{\chi}(n)+\frac{n}{2}\left(\ln \frac{r}{\pi}-\gamma\right)+\tau_{\chi}(n),
$$


where

$$
\begin{gathered}
S_{\chi}(n) \equiv-\sum_{j=1}^{n}\left(\begin{array}{c}
n \\
j
\end{array}\right) \frac{(-1)^{j-1}}{(j-1) !} \sum_{k=1}^{\infty} \frac{\Lambda(k)}{k} \bar{\chi}(k)(\ln k)^{j-1}, \\
=-\sum_{k=1}^{\infty} \frac{\Lambda(k)}{k} \bar{\chi}(k) L_{n-1}^{1}(\ln k), \\
\tau_{\chi}(n)=\sum_{j=2}^{n}\left(\begin{array}{c}
n \\
j
\end{array}\right)(-1)^{j}\left(1-2^{-j}\right) \zeta(j)-\frac{n}{2} \sum_{\ell=1}^{\infty} \frac{1}{\ell(2 \ell-1)} \quad \text { for } \quad \chi(-1)=1, \\
=\sum_{j=2}^{n}\left(\begin{array}{c}
n \\
j
\end{array}\right)(-1)^{j} 2^{-j} \zeta(j) \quad \text { for } \quad \chi(-1)=-1,
\end{gathered}
$$

and $L_{n}^{\alpha}$ is an associated Laguerre polynomial. We recall Eq. (25) of the text,

$$
\begin{aligned}
& \tau_{\chi}(n)=S_{1}-n \ln 2 \quad \text { if } \quad \chi(-1)=1 . \\
& =S_{0}-S_{1}, \quad \text { if } \quad \chi(-1)=-1 .
\end{aligned}
$$

where $S_{0}$ is defined in Eq. (A.15) of Appendix A. Therefore, by using the summation estimations presented in Appendix A, we obtain

$$
\begin{aligned}
\lambda_{\chi} \geq & S_{\chi}(n)+\frac{n}{2} \ln n+\frac{n}{2}\left(\ln \frac{r}{\pi}-1-2 \ln 2\right)+\frac{1}{2} \quad \text { for } \quad \chi(-1)=1, \\
& \geq S_{\chi}(n)+\frac{n}{2} \ln n+\frac{n}{2}\left(\ln \frac{r}{\pi}-1\right)+\frac{1}{2} \quad \text { for } \quad \chi(-1)=-1 .
\end{aligned}
$$

In accord with the discussion of the text, we conjecture that the sum $S_{\chi}$ is 'small'. By this we mean that $S_{\chi}(n)$ could be $O(n)$ and probably even $S_{\chi}$ is $O\left(n^{1 / 2+\epsilon}\right)$, for $\epsilon>0$. This could result from a near exponential amount of cancellation in this sum due to the phases present in the Dirichlet characters.

We next introduce the function

$$
\xi_{E}(s)=c_{E} N^{s / 2}(2 \pi)^{-s} \Gamma\left(s+\frac{1}{2}\right) L_{E}\left(s+\frac{1}{2}\right)
$$


where $L_{E}$ is the L-series associated with an elliptic curve $E$ over the rational numbers, $N$ is the conductor, and $c_{E}$ is a constant chosen so that $\xi_{E}(1)=1[7,30]$. The function of Eq. (E.8) is an entire function of order one and satisfies $\xi_{E}(s)=w \xi_{E}(1-s)$ where $w=(-1)^{r}$ with $r$ being the vanishing order of $\xi_{E}(s)$ at $s=1 / 2$.

We let

$$
\lambda_{E}(n)=\sum_{\rho}\left[1-\left(1-\frac{1}{\rho}\right)^{n}\right], \quad n \geq 1,
$$

where the sum is over all zeros $\rho$ of $\xi_{E}(s)$. All of these zeros lie on the critical line if and only if $[26] \lambda_{E}(n)>0$ for all $n=1,2, \ldots$

Now Li [26] has obtained the explicit formula

$$
\lambda_{E}(n)=S_{E}+n\left(\ln \frac{\sqrt{N}}{2 \pi}-\gamma\right)+n\left(-\frac{2}{3}+\sum_{\ell=1}^{\infty} \frac{3}{\ell(2 \ell+3)}\right)+\sum_{j=2}^{n}\left(\begin{array}{c}
n \\
j
\end{array}\right)(-1)^{j} \sum_{\ell=1}^{\infty} \frac{1}{(\ell+1 / 2)^{j}}
$$

where

$$
\begin{gathered}
S_{E}(n) \equiv-\sum_{j=1}^{n}\left(\begin{array}{c}
n \\
j
\end{array}\right) \frac{(-1)^{j-1}}{(j-1) !} \sum_{k=1}^{\infty} \frac{\Lambda(k)}{k^{3 / 2}} b(k)(\ln k)^{j-1}, \\
=-\sum_{k=1}^{\infty} \frac{\Lambda(k)}{k^{3 / 2}} b(k) L_{n-1}^{1}(\ln k) .
\end{gathered}
$$

In Eq. (E.11), $b\left(p^{k}\right)=a_{p}^{k}$ if $p \mid N$ and $b\left(p^{k}\right)=\alpha_{p}^{k}+\beta_{p}^{k}$ if $(p, N)=1$, where for each prime number $p, \alpha_{p}$ and $\beta_{p}$ are the roots of the equation $T^{2}-a_{p} T+p$ and the values of $a_{p}$ are connected with the reduction of $E$ at $p[26]$.

We recall Eq. (26) of the text, so that we may write

$$
\lambda_{E}(n)=S_{E}(n)+n\left(\ln \frac{\sqrt{N}}{2 \pi}-\gamma\right)+2\left(1-\frac{1}{3} \ln 2\right) n+S_{3}-S_{0}+2 n+(-1)^{n}-1,
$$


where the zeta function sum $S_{3}$ is defined in Eq. (A.18) of Appendix A (and see below, Eq. (E.15)). Then, by the results of Appendix A we obtain

$$
\lambda_{E}(n) \geq S_{E}(n)+n \ln n+n \ln \frac{\sqrt{N}}{2 \pi}+\left(3+\frac{4}{3} \ln 2\right) n-1 .
$$

Again, we conjecture that the sum $S_{E}$ is $O\left(n^{1 / 2+\epsilon}\right)$. The values of $a_{p}$ include 0 and \pm 1 , so that the values of $b\left(p^{k}\right)$ can either be zero or include significant sign alternation when $p \mid N$. Similarly, for $(p, N)=1$, the roots of $T^{2}-a_{p} T+p$ can include $\pm \sqrt{p}$ and $[ \pm 1 \pm \sqrt{1-4 p}] / 2$, giving various sign changes in $b\left(p^{k}\right)$. When $E$ has good reduction at $p,-2 \sqrt{p} \leq a_{p} \leq 2 \sqrt{p}$, so that it again appears that $b\left(p^{k}\right)$ can have significant changes in sign, possibly leading to much cancellation in $S_{E}$.

Concerning Eqs. (E.6) and (E.12) we record and briefly discuss some elementary summation results. We have

$$
\ln 2=\sum_{n=1}^{\infty} \frac{1}{2 n-1}-\frac{1}{2} \sum_{n=1}^{\infty} \frac{1}{n}
$$

giving

$$
\ln 2=\frac{4}{3}-3 \sum_{n=1}^{\infty} \frac{1}{2 n(2 n+3)}
$$

leading to

$$
-\frac{2}{3}+\sum_{n=1}^{\infty} \frac{3}{n(2 n+3)}=2(1-\ln 2) .
$$

In addition, we have

$$
\sum_{\ell=1}^{\infty} \frac{1}{(\ell+1 / 2)^{j}}=\sum_{m=1}^{\infty} \frac{2^{j}}{(2 m+1)^{j}}=\sum_{m=1}^{\infty}\left(\frac{2}{2 m+1}\right)^{j}+\sum_{m=1}^{\infty}\left(\frac{2}{2 m}\right)^{j}-\zeta(j)
$$




$$
\begin{aligned}
& =\sum_{k=3, \text { odd }}^{\infty}\left(\frac{2}{k}\right)^{j}+\sum_{k=2, \text { even }}^{\infty}\left(\frac{2}{k}\right)^{j}-\zeta(j) \\
& =\sum_{k=2}^{\infty}\left(\frac{2}{k}\right)^{j}-\zeta(j)=2^{j}[\zeta(j)-1]-\zeta(j) .
\end{aligned}
$$

This equation is a restatement of the relation between the Riemann zeta function and the Hurwitz zeta function $\zeta(s, a): \zeta(s)=\zeta(s, 1)=\left(2^{s}-1\right)^{-1} \zeta(s, 1 / 2)$.

The sum of Eq. (E.15) also has a close relation to $\psi^{(n)}(1 / 2)$, where $\psi^{(j)}$ is the polygamma function, because [16]

$$
\psi^{(n)}\left(\frac{1}{2}\right)=(-1)^{n+1} n ! \sum_{k=0}^{\infty} \frac{1}{(k+1 / 2)^{n+1}} .
$$

Then, with the use of Eq. (E.15), we have

$$
\psi^{(n)}\left(\frac{1}{2}\right)=(-1)^{n+1} n !\left(2^{n+1}-1\right) \zeta(n+1),
$$

which is the expected result. In general, we have $\psi^{(n)}(x)=(-1)^{n+1} n ! \zeta(n+1, x)$.

We may also write an integral representation for the polygamma function which is very useful for evaluating terms in explicit formulas for sums over zeros of zeta functions. By differentiating an integral representation for $\psi(z)+\gamma$, we have

$$
\psi^{(m-1)}(z)=(-1)^{m} \int_{0}^{\infty} \frac{e^{-z t} t^{m-1}}{1-e^{-t}} d t=\frac{(-1)^{m}}{2} \int_{0}^{\infty} \frac{t^{m-1} e^{-(z-1 / 2) t}}{\sinh (t / 2)} d t
$$

giving the specific values

$$
\begin{aligned}
& \psi^{(m-1)}\left(\frac{1}{2}\right)=(-1)^{m} 2^{m-1} \int_{0}^{\infty} \frac{y^{m-1}}{\sinh y} d y, \\
& \psi^{(m-1)}(1)=(-1)^{m} 2^{m-1} \int_{0}^{\infty} \frac{y^{m-1} e^{-y}}{\sinh y} d y,
\end{aligned}
$$


and

$$
\psi^{(m-1)}\left(\frac{3}{2}\right)=\frac{(-1)^{m}}{2} \int_{0}^{\infty} \frac{t^{m-1} e^{-t}}{\sinh (t / 2)} d t
$$




\section{Appendix EII: Explicit Formulas for Dirichlet and Hecke L-Functions}

Here we give alternative derivations of the very recent main results of $\mathrm{Li}$ [26], Theorems 1 and 2, of Ref. [26]. The procedure is very similar to the proof of Theorem 1 of the text. The Riemann zeta function case extends since the Dirichlet and Hecke L-functions also have product expansions over their zeros and have explicit forms of their logarithmic derivatives. These derivations also make it very apparent that certain polygamma constants are the source of the elementary sums described in the first part of this Appendix.

Due to the product expansion of $\xi(s, \chi)$, we have the formula

$$
\lambda_{\chi}(n)=\sum_{m=1}^{n}\left(\begin{array}{c}
n \\
m
\end{array}\right) \frac{1}{(m-1) !}\left[\frac{d^{m}}{d s^{m}} \ln \xi(s, \chi)\right]_{s=1} .
$$

This equation is the analog of Eq. (15) of the text or Eq. (G.5) of Appendix G for the Riemann zeta function case. From Eq. (E.1) we have

$$
\ln \xi(s, \chi)=-\frac{(s+a)}{2} \ln \left(\frac{\pi}{r}\right)+\ln \Gamma\left(\frac{s+a}{2}\right)+\ln L(s, \chi),
$$

giving

$$
\frac{d}{d s} \ln \xi(s, \chi)=\ln \left(\frac{r}{\pi}\right)+\frac{1}{2} \psi\left(\frac{s+a}{2}\right)-\sum_{n=1}^{\infty} \frac{\Lambda(n) \chi(n)}{n^{s}}, \quad \operatorname{Re} s>1,
$$

where $\psi=\Gamma^{\prime} / \Gamma$ is the digamma function and $\Lambda$ is the von Mangoldt function, such that $\Lambda(k)=\ln p$ when $k$ is a power of a prime and $\Lambda(k)=0$ otherwise. For $m \geq 2$, we then have

$$
[\ln \xi(s, \chi)]^{(m)}=\frac{1}{2^{m}} \psi^{(m-1)}\left(\frac{s+a}{2}\right)-(-1)^{m-1} \sum_{n=1}^{\infty} \frac{\Lambda(n) \chi(n)}{n^{s}} \ln ^{m-1} n,
$$


where $\psi^{(n)}$ is again the polygamma function. By taking the limit $s \rightarrow 1$ in Eq. (E.22) we then obtain the representation

$$
\begin{aligned}
\lambda_{\chi}(n)=\left[\ln \left(\frac{r}{\pi}\right)\right. & \left.+\psi\left(\frac{a+1}{2}\right)\right] \frac{n}{2}+\sum_{m=2}^{n}\left(\begin{array}{c}
n \\
m
\end{array}\right) \frac{1}{(m-1) !} 2^{-m} \psi^{(m-1)}\left(\frac{a+1}{2}\right) \\
& -\sum_{m=1}^{n}\left(\begin{array}{c}
n \\
m
\end{array}\right) \frac{(-1)^{m-1}}{(m-1) !} \sum_{n=1}^{\infty} \frac{\Lambda(n) \chi(n)}{n} \ln ^{m-1} n,
\end{aligned}
$$

where $\psi(1 / 2)=-\gamma-2 \ln 2, \psi(1)=-\gamma, \gamma$ is the Euler constant, $\psi^{(m-1)}(1)=$ $(-1)^{m}(m-1) ! \zeta(m)$, and $\psi^{(m-1)}(1 / 2)$ is given in Eq. (E.16b). The infinite series in the sum $S_{\chi}(n)$ is convergent by the prime number theorem for arithmetic progressions $[26,12]$. We have therefore obtained the result Eq. (E.3).

Similarly, due to the product expansion of $\xi_{E}(s)$, we have the formula

$$
\lambda_{E}(n)=\sum_{m=1}^{n}\left(\begin{array}{l}
n \\
m
\end{array}\right) \frac{1}{(m-1) !}\left[\frac{d^{m}}{d s^{m}} \ln \xi_{E}(s)\right]_{s=1}
$$

where from Eq. (E.8) we have

$$
\ln \xi_{E}(s)=\ln c_{E}+\frac{s}{2} \ln N-s \ln 2 \pi+\ln \Gamma\left(s+\frac{1}{2}\right)+\ln L_{E}\left(s+\frac{1}{2}\right)
$$

and

$$
\frac{d}{d s} \ln \xi_{E}(s)=\frac{1}{2} \ln N-\ln 2 \pi+\psi\left(s+\frac{1}{2}\right)-\sum_{n=1}^{\infty} \frac{\Lambda(n) b(n)}{n^{s+1 / 2}}, \quad \operatorname{Re} s>1
$$

where $b(n)$ is discussed in the first part of this Appendix. For $m \geq 2$, we then have

$$
\left[\ln \xi_{E}(s)\right]^{(m)}=\psi^{(m-1)}\left(s+\frac{1}{2}\right)-(-1)^{m-1} \sum_{n=1}^{\infty} \frac{\Lambda(n) b(n)}{n^{s+1 / 2}} \ln ^{m-1} n
$$


By taking the limit $s \rightarrow 1$ in Eq. (E.27) we then obtain the representation

$$
\begin{aligned}
\lambda_{E}(n)=[ & \left.\ln \left(\frac{\sqrt{N}}{2 \pi}\right)+\psi\left(\frac{3}{2}\right)\right] n+\sum_{m=2}^{n}\left(\begin{array}{c}
n \\
m
\end{array}\right) \frac{1}{(m-1) !} \psi^{(m-1)}\left(\frac{3}{2}\right) \\
& -\sum_{m=1}^{n}\left(\begin{array}{c}
n \\
m
\end{array}\right) \frac{(-1)^{m-1}}{(m-1) !} \sum_{n=1}^{\infty} \frac{\Lambda(n) b(n)}{n^{3 / 2}} \ln ^{m-1} n,
\end{aligned}
$$

where $\psi(3 / 2)=2(1-\ln 2)-\gamma$ and $\psi^{(m-1)}(3 / 2)=(-1)^{m}(m-1) !\left[2^{m}(\zeta(m)-1)-\zeta(m)\right]$, giving the result Eq. (E.12).

Finally, we consider the case of the Dedekind zeta function $\zeta_{k}$, for which we need to introduce some additional notation. We let $k$ be an algebraic number field with $r_{1}$ real places, $r_{2}$ imaginary places, and degree $\tilde{n}=r_{1}+2 r_{2}$. The zeta function $\zeta_{k}$ has the product expansion $\zeta_{k}(s)=\prod_{p}\left(1-N p^{-s}\right)^{-1}$ for $\operatorname{Re} s>1$, where the product is taken over all finite prime divisors of $k$. We put $G_{1}(s)=\pi^{-s / 2} \Gamma(s / 2)$ and $G_{2}(s)=(2 \pi)^{1-s} \Gamma(s)$, so that obviously $G_{1}(1)=G_{2}(1)=1$. Then the function

$$
Z_{k}(s) \equiv G_{1}^{r_{1}}(s) G_{2}^{r_{2}}(s) \zeta_{k}(s)
$$

satisfies the functional equation $Z_{k}(s)=\left|d_{k}\right|^{1 / 2-s} Z_{k}(1-s)$, where $d_{k}$ is the discriminant of $k$.

We let $c_{k}=2^{r_{1}}(2 \pi)^{r_{2}} h R / e$, where $h, R$, and $e$ are respectively the number of ideal classes of $k$, the regulator of $k$, and the number of roots of unity in $k$. With

$$
\xi_{k}(s) \equiv c_{k}^{-1} s(s-1)\left|d_{k}\right|^{s / 2} Z_{k}(s)
$$

this function is entire and has $\xi_{k}(0)=1[40,32]$. We first present a motivation that an explicit formula analogous to that for $\lambda_{E}$ and $\lambda_{\chi}$ exists, and then develop the 
corresponding explicit formula, putting

$$
\lambda_{n}=\sum_{m=1}^{n}\left(\begin{array}{c}
n \\
m
\end{array}\right) \frac{1}{(m-1) !}\left[\frac{d^{m}}{d s^{m}} \ln \xi_{k}(s)\right]_{s=1} .
$$

From Eqs. (E.29) and (E.30) we have

$$
\begin{gathered}
\ln \xi_{k}(s)=-\ln c_{k}+\ln s+\ln (s-1)+\frac{s}{2} \ln \left|d_{k}\right|+r_{1}\left[-\frac{s}{2} \ln \pi-\ln \Gamma\left(\frac{s}{2}\right)\right] \\
+r_{2}[(1-s) \ln (2 \pi)+\ln \Gamma(s)]+\ln \zeta_{k}(s),
\end{gathered}
$$

and

$\frac{d}{d s} \ln \xi_{k}(s)=\frac{1}{s}+\frac{1}{s-1}+\frac{1}{2} \ln \left|d_{k}\right|+\frac{r_{1}}{2}\left[-\ln \pi+\psi\left(\frac{s}{2}\right)\right]+r_{2}[-\ln (2 \pi)+\psi(s)]+\frac{\zeta_{k}^{\prime}(s)}{\zeta_{k}(s)}, \quad \operatorname{Re} s>1$,

where [34]

$$
\frac{\zeta_{k}^{\prime}(s)}{\zeta_{k}(s)}=-\sum_{p} \sum_{m=1}^{\infty} \frac{\ln N p}{N p^{m s}}, \quad \operatorname{Re} s>1 .
$$

In Eq. (E.34), $p$ runs over the prime ideals of $k$ and $N$ represents the norm.

For $m \geq 2$, we then have

$$
\begin{gathered}
\frac{d^{m}}{d s^{m}} \ln \xi_{k}(s)=\frac{(-1)^{m}(m-1) !}{s^{m-1}}+\frac{(-1)^{m}(m-1) !}{(s-1)^{m-1}}+\frac{r_{1}}{2^{m}} \psi^{(m-1)}\left(\frac{s}{2}\right)+r_{2} \psi^{(m-1)}(s) \\
+\frac{d^{m-1}}{d s^{m-1}}\left[\frac{\zeta_{k}^{\prime}(s)}{\zeta_{k}(s)}\right], \quad \text { Re } s>1,
\end{gathered}
$$

where the evaluation of the first, third and fourth terms on the right side of Eq. (E.35) at $s=1$ gives the contribution to $\lambda_{n}$ of

$$
\lambda_{n}^{(\psi)}=\sum_{m=2}^{n}(-1)^{m}\left(\begin{array}{c}
n \\
m
\end{array}\right)\left\{1+\left[\left(1-2^{-m}\right) r_{1}+r_{2}\right] \zeta(m)\right\} .
$$


The evaluation of all of the terms on the right side of Eq. (E.33) but the second and last at $s=1$ gives to $\lambda_{n}$ the contribution $n\left[1+\frac{1}{2} \ln \left|d_{k}\right|-\frac{\tilde{n}}{2}(\ln \pi+\gamma)-\left(r_{1}+r_{2}\right) \ln 2\right]$. With the aid of Eq. (E.34) we have

$$
\frac{d^{m-1}}{d s^{m-1}}\left[\frac{\zeta_{k}^{\prime}(s)}{\zeta_{k}(s)}\right]=-\sum_{p} \sum_{\ell=1}^{\infty}(-1)^{m-1} \ell^{m-1} \frac{\ln ^{m} N p}{N p^{\ell s}}, \quad \text { Re } s>1 .
$$

Taking the limit $s \rightarrow 1$ in Eqs. (E.33)-(E.35) and (E.37) should yield the final explicit representation for $\lambda_{n}$, subject to justification of the convergence of the resulting series. 


\section{Appendix F: Further Riemann zeta function sum estimations}

As a generalization of sums such as $S_{1}$ of Eq. (16) of the text and $S_{0}$ of Eq. (A.15) of Appendix A, we consider here sums of the form

$$
S_{\nu}(\kappa, n) \equiv \sum_{m=\nu+2}^{n}(-1)^{m}\left(\begin{array}{l}
n \\
m
\end{array}\right) \kappa^{m} \zeta(m-\nu),
$$

where $\nu+2 \leq n$, which can be extended to $|\operatorname{Re} \nu|+2 \leq n$. In Eq. (F.1) we have introduced both the positive multiplier $\kappa$ and shift $\nu$. The special cases of Eq. (F.1) of direct interest to this paper are $\nu=0$ with $\kappa=1$ or $\kappa=2^{ \pm 1}$. We are interested to both reformulate the sum $S_{\nu}$ and to obtain a lower bound for it.

If we reorder the two sums in Eq. (F.1), the inner sum takes the form

$\sum_{m=\nu+2}^{n}(-1)^{m}\left(\begin{array}{l}n \\ m\end{array}\right) \frac{\kappa^{m}}{j^{m-\nu}}=(-1)^{\nu} \frac{\kappa^{\nu+2} n !}{j^{2}} \sum_{m=0}^{n-\nu-2} \frac{(1)_{m}}{(n-m-\nu-2) !(m+\nu+2) !} \frac{1}{m !}\left(\frac{\kappa}{j}\right)^{m}$.

If we use the relations $(m+\nu+2) !=(\nu+2) !(\nu+3)_{m}$ and $(n-m-\nu-2) !=$ $(n-\nu-2) ! /(2+\nu-n)_{m}$, where the Pochhammer symbol $(z)_{n}=\Gamma(z+n) / \Gamma(z)$, we obtain the terminating hypergeometric form

$$
\sum_{m=\nu+2}^{n}(-1)^{m}\left(\begin{array}{c}
n \\
m
\end{array}\right) \frac{\kappa^{m}}{j^{m-\nu}}=(-1)^{\nu}\left(\begin{array}{c}
n \\
\nu+2
\end{array}\right) \frac{\kappa^{\nu+2}}{j^{2}}{ }_{2} F_{1}(1,2-n+\nu ; \nu+3 ; \kappa / j),
$$

giving

$$
S_{\nu}(\kappa, n)=(-1)^{\nu}\left(\begin{array}{c}
n \\
\nu+2
\end{array}\right) \kappa^{\nu+2} \sum_{j=1}^{\infty} \frac{1}{j^{2}}{ }_{2} F_{1}(1,2-n+\nu ; \nu+3 ; \kappa / j) .
$$

We now define the comparison integral

$$
I_{\nu}(\kappa, n) \equiv(-1)^{\nu}\left(\begin{array}{c}
n \\
\nu+2
\end{array}\right) \kappa^{\nu+2} \int_{1}^{\infty} \frac{1}{j^{2}}{ }_{2} F_{1}(1,2-n+\nu ; \nu+3 ; \kappa / j) d j
$$


With the change of variable $v=1 / j$ the integration is easily accomplished [16] in terms of the generalized hypergeometric function ${ }_{p} F_{q}[3]$ :

$$
I_{\nu}(\kappa, n)=(-1)^{\nu}\left(\begin{array}{c}
n \\
\nu+2
\end{array}\right) \kappa_{3}^{\nu+2} F_{2}(1,1, \nu+2-n ; 2, \nu+3 ; \kappa) .
$$

Equation (F.6) is simply the result of term-by-term integration and the fact that $(1)_{k} /(2)_{k}=1 /(k+1)$. The relation $S_{\nu}(\kappa, n) \geq I_{\nu}(\kappa, n)$ then yields a family of inequalities.

When $\kappa$ is unity we have the reduction $\psi(z)=(z-1){ }_{3} F_{2}(1,1,2-z ; 2,2 ; 1)-\gamma$ and therefore

$$
I_{\nu}(1, n)=(-1)^{\nu}\left(\begin{array}{c}
n \\
\nu+1
\end{array}\right)\left[\psi(n+1)+\gamma-H_{\nu+1}\right]
$$

where $\psi$ is the digamma function and $H_{n}=\sum_{k=1}^{n} 1 / k$ is the $n$th harmonic number [10], which can also be written as

$$
I_{\nu}(1, n)=(-1)^{\nu}\left(\begin{array}{c}
n \\
\nu+1
\end{array}\right)\left[H_{n}-H_{\nu+1}\right]
$$

For $\nu=0$, this gives the result of Eq. (A.17). An alternative form of $I_{\nu}(1, n)$ can be obtained by applying Theorem 1 of Ref. [36]:

$$
I_{\nu}(1, n)=(-1)^{\nu}\left(\begin{array}{c}
n \\
\nu+1
\end{array}\right)\left[\psi(n-\nu)-\psi(\nu+2)-\sum_{k=1}^{\nu+1} \frac{(-\nu-1)_{k}}{k(n-\nu)_{k}}\right]
$$

valid for $n>0$ and integral $\nu \geq-1$. The relations above at unit argument can also be looked upon as special cases of [28]

$$
{ }_{3} F_{2}(1,1, \nu+1 ; 2, \lambda+1 ; 1)=\frac{\lambda}{\nu}[\psi(\lambda)-\psi(\lambda-\nu)], \quad \nu \neq 0, \operatorname{Re}(\lambda-\nu)>0
$$




$$
=\lambda \psi^{\prime}(\lambda), \quad \nu=0, \quad \operatorname{Re}(\lambda)>0,
$$

i.e.,

$$
\sum_{n=1}^{\infty} \frac{(\nu)_{n}}{n(\lambda)_{n}}=\psi(\lambda)-\psi(\lambda-\nu), \quad \operatorname{Re}(\lambda-\nu)>0, \quad \lambda \neq 0,-1,-2, \ldots
$$

For $\kappa=1 / 2$ we obtain

$$
I_{\nu}(1 / 2, n)=(-1)^{\nu}\left(\begin{array}{c}
n \\
\nu+2
\end{array}\right) \frac{1}{2^{\nu+2}}{ }_{3} F_{2}(1,1, \nu+2-n ; 2, \nu+3 ; 1 / 2),
$$

which for $\nu=0$ is in agreement with $I_{0}-I_{1}$, where $I_{1}$ is given in Eq. (24) of the text. For $\kappa=2$ we obtain

$$
I_{\nu}(2, n)=(-1)^{\nu}\left(\begin{array}{c}
n \\
\nu+2
\end{array}\right) 2^{\nu+2}{ }_{3} F_{2}(1,1, \nu+2-n ; 2, \nu+3 ; 2),
$$

which is in agreement with $I_{3}$ of Eq. (A.22) of Appendix A when $\nu=0$. 


\section{Appendix G: Alternative representation of Li's $\lambda_{j}$ 's}

Here we extend Theorem 1, based upon an expansion of the logarithmic derivative of the Riemann zeta function with a larger radius of convergence than Eq. (12) of the text. We demonstrate the following representation,

$$
\begin{gathered}
\lambda_{n}=-\sum_{m=1}^{n}\left(\begin{array}{c}
n \\
m
\end{array}\right) \eta_{m-1}^{(12)}+7-\left[\left(\frac{2}{3}\right)^{n}+\left(\frac{4}{5}\right)^{n}+\left(\frac{6}{7}\right)^{n}+\left(\frac{8}{9}\right)^{n}+\left(\frac{10}{11}\right)^{n}+\left(\frac{12}{13}\right)^{n}\right] \\
+\sum_{m=2}^{n}(-1)^{m}\left(\begin{array}{c}
n \\
m
\end{array}\right)\left(1-2^{-m}\right) \zeta(m)-\frac{n}{2}(\gamma+\ln \pi+2 \ln 2),
\end{gathered}
$$

where we currently do not have an arithmetic interpretation of the constants $\eta_{j}^{(12)}$.

From the expansion around $s=1$ of the logarithmic derivative of the zeta function,

$$
\frac{\zeta^{\prime}(s)}{\zeta(s)}=-\frac{1}{(s-1)}+\sum_{j=1}^{6} \frac{1}{(s+2 j)}-\sum_{p=0}^{\infty} \eta_{p}^{(12)}(s-1)^{p},
$$

we have

$$
\ln \zeta(s)=-\ln (s-1)+\sum_{j=1}^{6} \ln (s+2 j)-\sum_{p=1}^{\infty} \frac{\eta_{p-1}^{(12)}}{p}(s-1)^{p}+\text { constant },
$$

giving

$\ln \xi(s)=-\ln 2+\ln s-\frac{s}{2} \ln \pi+\ln \Gamma\left(\frac{s}{2}\right)+\mathrm{constant}+\sum_{j=1}^{6} \ln (s+2 j)-\sum_{p=1}^{\infty} \frac{\eta_{p-1}^{(12)}}{p}(s-1)^{p}$.

With the expansion (G.2) the radius of convergence has been increased to 13 (see Figure 1), as we have included the contribution of all trivial zeros of $\zeta$ prior to the encounter with the first complex zero $\rho_{1}$. We next evaluate

$$
\lambda_{n}=\sum_{m=1}^{n}\left(\begin{array}{c}
n \\
m
\end{array}\right) \frac{1}{(m-1) !}\left[\frac{d^{m}}{d s^{m}} \ln \xi(s)\right]_{s=1},
$$


using again the special values $\psi(1 / 2)=-\gamma-2 \ln 2$ and $\psi^{(n)}(1 / 2)=(-1)^{n+1} n !\left(2^{n+1}-\right.$ 1) $\zeta(n+1)$ for $n \geq 1$, where $\psi=\Gamma^{\prime} / \Gamma$ is the digamma function and $\psi^{(j)}$ is the polygamma function. Recalling the relations

$$
\frac{d^{j}}{d s^{j}} \ln (s+2 k)=-\frac{(-1)^{j}(j-1) !}{(s+2 k)^{j}}, \quad j \geq 1,
$$

and $\left(d^{j} / d s^{j}\right)(s-1)^{k}=0$ for $k<j$, and the sum

$$
\sum_{m=1}^{n}\left(\begin{array}{l}
n \\
m
\end{array}\right)\left(-\frac{1}{k}\right)^{m}=-1+\left(\frac{k-1}{k}\right)^{n},
$$

we find Eq. (G.1). The constant term of $6=7-1$ in Eq. (G.1) serves as a count of the number of trivial zeros of $\zeta$ accounted for in the expansion (G.2) while the additional explicit negative terms beyond Eq. (10) of the text appearing there are exponentially decreasing with $n$. We have developed in Eq. (G.2) an expansion with coefficients $\eta_{j}^{(12)}$ whose magnitudes increase no faster than $1 / 13^{j}$ for large $j$.

By using Eq. (20) for $S_{1}$, an extension of the Corollary of the text is

\section{Corollary G1}

$$
\begin{gathered}
\lambda_{n} \geq \frac{n}{2} \ln n-(1+\ln \pi+2 \ln 2) \frac{n}{2}+\frac{15}{2} \\
-\left[\left(\frac{2}{3}\right)^{n}+\left(\frac{4}{5}\right)^{n}+\left(\frac{6}{7}\right)^{n}+\left(\frac{8}{9}\right)^{n}+\left(\frac{10}{11}\right)^{n}+\left(\frac{12}{13}\right)^{n}\right]-\left|S_{2}^{(12)}\right|,
\end{gathered}
$$

where we have put $S_{2}^{(12)} \equiv-\sum_{m=1}^{n}\left(\begin{array}{c}n \\ m\end{array}\right) \eta_{m-1}^{(12)}$.

In developing expansions such as

$\zeta(s->-2 k)=\zeta^{\prime}(-2 k)(s+2 k)+\frac{1}{2} \zeta^{\prime \prime}(-2 k)(s+2 k)^{2}+\frac{1}{6} \zeta^{\prime \prime \prime}(-2 k)(s+2 k)^{3}+O\left[(s+2 k)^{4}\right], \quad k \geq 1$, 
and

$$
\frac{\zeta^{\prime}(s)}{\zeta(s)}=\frac{1}{(s+2 k)}+\frac{\zeta^{\prime \prime}(-2 k)}{2 \zeta^{\prime}(-2 k)}+\left[-\frac{\left[\zeta^{\prime \prime}(-2 k)\right]^{2}}{4\left[\zeta^{\prime}(-2 k)\right]^{2}}+\frac{\zeta^{\prime \prime \prime}(-2 k)}{3 \zeta^{\prime}(-2 k)}\right](s+2 k)+O\left[(s+2 k)^{2}\right],
$$

it is useful to have the derivatives

$$
\zeta^{\prime}(-2 n)=(-1)^{n} \frac{(2 n) !}{2(2 \pi)^{2 n}} \zeta(2 n+1), \quad n \geq 1
$$

and

$\zeta^{\prime \prime}(-2 n)=(-1)^{n} \frac{(2 n) !}{2(2 \pi)^{2 n}}\left\{\left[\ln \left(4 \pi^{2}\right)-2 \psi(2 n)-\frac{1}{n}\right] \zeta(2 n+1)-2 \zeta^{\prime}(2 n+1)\right\}, \quad n \geq 1$

which follow easily by differentiating the functional equation for $\zeta(s)$ and putting $s=2 n+1$.

We note in passing the numerical value of the constant

$$
\frac{1}{2} \sum_{k=1}^{6} \frac{\zeta^{\prime \prime}(-2 k)}{\zeta^{\prime}(-2 k)}=-\frac{81959}{5544}+6(\gamma+\ln 2 \pi)-\sum_{k=1}^{6} \frac{\zeta^{\prime}(2 k+1)}{\zeta(2 k+1)} \simeq-0.0926073 .
$$

We see from Eq. (G.2) that the value of $\eta_{0}^{(12)}$ is given by

$$
\eta_{0}^{(12)}=\gamma-\sum_{k=1}^{6} \frac{1}{2 k+1} \simeq 0.377918
$$

Therefore we can modify Eq. (G.8) to

\section{Corollary G2}

$$
\begin{gathered}
\lambda_{n} \geq \frac{n}{2} \ln n-(1+\ln \pi+2 \ln 2) \frac{n}{2}+\frac{15}{2} \\
-\left[\left(\frac{2}{3}\right)^{n}+\left(\frac{4}{5}\right)^{n}+\left(\frac{6}{7}\right)^{n}+\left(\frac{8}{9}\right)^{n}+\left(\frac{10}{11}\right)^{n}+\left(\frac{12}{13}\right)^{n}\right]-\left|\eta_{0}^{(12)}\right| n-\left|\sum_{m=2}^{n}\left(\begin{array}{c}
n \\
m
\end{array}\right) \eta_{m-1}^{(12)}\right| .
\end{gathered}
$$




\section{Appendix H: On derivatives of the Riemann zeta function}

Here we capture various formulas for integer order derivatives of the zeta function. We anticipate that these could be useful in further development of the discrete

moment problem for the coefficients $\eta_{j}$ of Eqs. (10)-(14) and Eq. (41) and $\eta_{j}^{(12)}$ of Eqs. (G.1)-(G.4) of Appendix G.

We first note that the functional equation for $\zeta$, along with the evaluation $\zeta(1-$ $2 n)=-B_{2 n} / 2 n$ for $n \geq 1$, where $B_{n}$ are Bernoulli numbers, yields

$$
\zeta^{\prime}(-1)=\frac{\zeta^{\prime}(2)}{2 \pi^{2}}+\frac{1}{12}(\ln 2 \pi+\gamma),
$$

and

$$
\begin{gathered}
\zeta^{\prime \prime}(-1)=\frac{1}{6}\left(\frac{\pi^{2}}{8}-\frac{\ln ^{2} 2}{2}\right)+\left[(1-\gamma) \frac{\pi^{2}}{6}+\zeta^{\prime}(2)\right] \frac{\ln 2}{\pi^{2}}-\frac{(1-\gamma)}{\pi^{2}} \zeta^{\prime}(2)-\frac{1}{2 \pi^{2}} \zeta^{\prime \prime}(2) \\
-\frac{1}{12}\left[-1+(1-\gamma)^{2}+\frac{\pi^{2}}{6}\right]+\frac{1}{12} \ln ^{2} \pi+2 \ln \pi \zeta^{\prime}(-1),
\end{gathered}
$$

where $\gamma$ is the Euler constant, and this can be continued to higher order derivatives. The derived functional equation upon which Eqs. (H.1) and (H.2) are based is

$$
\begin{gathered}
2^{1-s} \Gamma(s) \zeta(s) \cos \left(\frac{\pi}{2} s\right)[\psi(s)-\ln 2]+2^{1-s} \Gamma(s) \zeta^{\prime}(s) \cos \left(\frac{\pi}{2} s\right) \\
-\frac{\pi}{2} 2^{1-s} \Gamma(s) \zeta(s) \sin \left(\frac{\pi}{2} s\right)=\pi^{s} \ln \pi \zeta(1-s)-\pi^{s} \zeta^{\prime}(1-s) .
\end{gathered}
$$

Now Elizalde [14] has given an expression for $\zeta^{\prime}(-m, q)$, where $\zeta(z, q)$ is the Hurwitz zeta function, valid for any negative integer value of $z$. We state here special cases for $q=1$ :

$$
\zeta^{\prime}(-1)=-\frac{1}{6}-\frac{1}{2} \sum_{k=1}^{\infty} \frac{B_{2 k+2}}{k(2 k+1)(2 k+2)}
$$




$$
\zeta^{\prime}(-2)=-\frac{1}{36}-\sum_{k=1}^{\infty} \frac{B_{2 k+2}}{(2 k-1) k(2 k+1)(2 k+2)}
$$

and

$$
\zeta^{\prime}(-k)=-\frac{1}{(k+1)^{2}}-\sum_{\ell=1}^{\infty} \frac{(-1)^{\ell}(2 \ell-1) !}{2^{2 \ell-1} \pi^{2 \ell}}\left[\sum_{r=0}^{\min (2 \ell-2, k)}\left(\begin{array}{l}
k \\
r
\end{array}\right) \frac{(-1)^{r}}{(2 \ell-r-1)}\right] \zeta(2 \ell) .
$$

This means that we also have explicit expressions for $\zeta^{\prime}(1+k)$. In particular Eqs. (H.1) and (H.4) or (H.6) yield an explicit form for $\zeta^{\prime}(2)$.

On the other hand, we can employ the integral representation, Eq. (38) of the text, to at least partially yield explicit values of the zeta derivatives for $\operatorname{Re} s>1$. Other integral representation could be used for Re $s>0$, but Eq. (38) serves for illustration. In the following, $\psi$ denotes the digamma function and $\psi^{(j)}$ the polygamma function, as usual.

We have

$$
\begin{gathered}
\zeta^{\prime}(s)=-\psi(s) \zeta(s)+\frac{1}{\Gamma(s)} \int_{0}^{\infty} \frac{t^{s-1} \ln t}{e^{t}-1} d t \\
\zeta^{\prime \prime}(s)=-\psi^{\prime}(s) \zeta(s)+\psi^{2}(s) \zeta(s)-2 \frac{\psi(s)}{\Gamma(s)} \int_{0}^{\infty} \frac{t^{s-1} \ln t}{e^{t}-1} d t+\frac{1}{\Gamma(s)} \int_{0}^{\infty} \frac{t^{s-1} \ln ^{2} t}{e^{t}-1} d t
\end{gathered}
$$

and therefore

$$
\frac{\zeta^{\prime}(s)}{\zeta(s)}=-\psi(s)+\frac{1}{\Gamma(s) \zeta(s)} \int_{0}^{\infty} \frac{t^{s-1} \ln t}{e^{t}-1} d t
$$

and

$$
\left[\frac{\zeta^{\prime}(s)}{\zeta(s)}\right]^{\prime}=-\psi^{\prime}(s)+\frac{1}{\Gamma(s) \zeta(s)}\left\{\int_{0}^{\infty} \frac{t^{s-1} \ln ^{2} t}{e^{t}-1} d t-\frac{1}{\Gamma(s) \zeta(s)}\left[\int_{0}^{\infty} \frac{t^{s-1} \ln t}{e^{t}-1} d t\right]^{2}\right\} .
$$


This process can be continued,

$$
\begin{gathered}
{\left[\frac{\zeta^{\prime}(s)}{\zeta(s)}\right]^{\prime \prime}=-\psi^{\prime \prime}(s)+\psi^{3}(s)-\psi(s) \psi^{\prime}(s)-\psi^{\prime}(s) \frac{\zeta^{\prime}(s)}{\zeta(s)}+\psi^{2}(s) \frac{\zeta^{\prime}(s)}{\zeta(s)}} \\
+\frac{1}{\Gamma(s) \zeta(s)}\left[\psi^{\prime}(s)-\psi^{2}(s)\right] \int_{0}^{\infty} \frac{t^{s-1} \ln t}{e^{t}-1} d t-\frac{3}{\Gamma^{2}(s) \zeta^{2}(s)} \int_{0}^{\infty} \frac{t^{s-1} \ln t}{e^{t}-1} d t \int_{0}^{\infty} \frac{t^{s-1} \ln ^{2} t}{e^{t}-1} d t \\
+\frac{1}{\Gamma(s) \zeta(s)} \int_{0}^{\infty} \frac{t^{s-1} \ln ^{3} t}{e^{t}-1} d t+\frac{2}{\Gamma^{3}(s) \zeta^{3}(s)}\left[\int_{0}^{\infty} \frac{t^{s-1} \ln t}{e^{t}-1} d t\right]^{3},
\end{gathered}
$$

and we note that

$$
-\psi^{(j)}(2)=(-1)^{j} j ![\zeta(j+1)-1] .
$$

Following on Eq. (H.7) we have

$$
\zeta^{(j+1)}(s)=-\sum_{m=0}^{j}\left(\begin{array}{c}
j \\
m
\end{array}\right) \psi^{(j-m)}(s) \zeta^{(m)}(s)+\sum_{m=0}^{j}\left(\begin{array}{c}
j \\
m
\end{array}\right)\left[\left(\frac{d}{d s}\right)^{m} \frac{1}{\Gamma(s)}\right] \int_{0}^{\infty} \frac{t^{s-1} \ln ^{j-m+1} t}{e^{t}-1} d t .
$$

The relations of this Appendix can be developed much more for application to Eq. (41) of the text or elsewhere. 


\section{Appendix I: A digamma function integral and a Mellin transform}

Here we consider the integral

$$
I(\lambda)=\int_{1}^{\infty} \frac{\left(1-x^{-\lambda}\right)}{x\left(x^{2}-1\right)} d x
$$

of Eq. (3.4) of Ref. [6] and evaluate it in two different ways from tabulated results [16]. In Ref. [6] the asymptotic behaviour of this integral for large $\lambda$ was of interest

for determining a certain limit denoted by the PF operation [Eq. (3.2) there]. We also note a polynomial of Ref. [6] that can be written as a terminating confluent hypergeometric series. This associated Laguerre polynomial was used in calculating forward and inverse Mellin transforms and we give alternative transforms.

With the change of variable $v(x)=x^{-1}$ in Eq. (I.1) we have

$$
I(\lambda)=\int_{0}^{\infty} \frac{v\left(1-v^{\lambda}\right)}{\left(1-v^{2}\right)} d v .
$$

From Ref. [16] we then obtain

$$
I(\lambda)=\frac{1}{2}\left[\psi\left(\frac{\lambda}{2}+1\right)+\gamma\right]=\frac{1}{2}\left[\psi\left(\frac{\lambda}{2}\right)+\gamma+\frac{2}{\lambda}\right], \quad \lambda>-2,
$$

where $\psi=\Gamma^{\prime} / \Gamma$ is the digamma function. On the other hand, we may employ a partial fractional decomposition in Eq. (I.2) and another tabulated result [16] so that $I(\lambda)=\frac{1}{2} \int_{0}^{1}\left(1-v^{\lambda}\right)\left[\frac{1}{(1-v)}-\frac{1}{(1+v)}\right] d v=\frac{1}{2}\left[\psi(\lambda+1)+\gamma-\ln 2+\int_{0}^{1} \frac{v^{\lambda}}{1+v} d v\right]$,

where the last integral is given by [16]

$$
\beta(\lambda+1)=\frac{1}{2}\left[\psi\left(\frac{\lambda}{2}+1\right)-\psi\left(\frac{\lambda+1}{2}\right)\right] .
$$


Use of the doubling formula $\psi[(\lambda+1) / 2]=2 \psi(\lambda)-\psi(\lambda / 2)-2 \ln 2$ then again yields Eq. (I.3). In Appendix A we have additionally given many inequalities for the digamma function.

The polynomial

$$
P_{n}(x)=\sum_{j=1}^{n}\left(\begin{array}{l}
n \\
j
\end{array}\right) \frac{x^{j-1}}{(j-1) !}
$$

was used in Ref. [6] in connection with computing Mellin transforms. By using the relations

$$
\left(\begin{array}{l}
n \\
j
\end{array}\right)=\frac{(-1)^{j}(-n)_{j}}{j !} \quad \text { and } \quad(-n)_{j+1}=-n(1-n)_{j},
$$

where $(.)_{n}$ is the Pochhammer symbol, this polynomial can be written as a terminating confluent hypergeometric series: $P_{n}(x)=n_{1} F_{1}(1-n ; 2 ;-x)$. In particular, a certain Mellin transform involving $P_{n}$ converts to a Laplace transform:

$$
\begin{gathered}
\int_{0}^{1} P_{n}(\ln x) x^{s-1} d x=\int_{0}^{\infty} P_{n}(-u) e^{-s u} d u=n \int_{0}^{\infty}{ }_{1} F_{1}(1-n ; 2 ; u) e^{-s u} d u \\
=\frac{n}{s} F\left(1-n, 1 ; 2 ; \frac{1}{s}\right)=1-\left(1-\frac{1}{s}\right)^{n},
\end{gathered}
$$

where $F$ is the Gauss hypergeometric function [16], which is the expected result [6]. In obtaining Eq. (I.8) we have used the reduction [16]

$$
F\left(1-n, 1 ; 2 ;-\frac{z}{t}\right)=\frac{(t+z)^{n}-t^{n}}{n z t^{n-1}} .
$$

Another useful point of view of the particular polynomial (I.6) is afforded by the theory of Laguerre polynomials $L_{n}$. This family is orthogonal on the interval $[0, \infty)$ with decaying exponential weight function. We have the relations

$$
P_{n}(-x)=-\frac{d L_{n}(x)}{d x}=L_{n-1}^{1}(x),
$$


where $L_{n}^{\alpha}$ is an associated Laguerre polynomial. In addition, the recursion relations satisfied by the Laguerre polynomials [16] give

$$
P_{n}(-x)=-\frac{n}{x}\left[L_{n}(x)-L_{n-1}(x)\right]=-\frac{(n+1)}{x} L_{n+1}(x)+\frac{(n+1-x)}{x} L_{n}(x) .
$$

Then one can recast the important Mellin transform-inverse transform pair of Eq. (I.8):

$$
\int_{0}^{\infty} P_{n}(-u) e^{-s u} d u=-\int_{0}^{\infty} \frac{d L_{n}(u)}{d u} e^{-s u} d u=1-\left(1-\frac{1}{s}\right)^{n}, \quad \operatorname{Re} s>0 .
$$

In obtaining this equation, one can use integration by parts, the Laplace transform of $L_{n}$ [16], and the property $L_{n}(0)=1$. All these relations are consistent with the connection $L_{n-1}^{1}(x)=n_{1} F_{1}(1-n ; 2 ; x)$ and the derivative property of the confluent hypergeometric function. 


\section{Appendix J: Regarding Conjectures 1-3}

We present here plausibility arguments in possible support of our conjectures concerning the detailed behaviour of the sequences $\left\{\sigma_{k}\right\}$ and $\left\{\lambda_{j}\right\}$. For this discussion, we let $N(T)$ be the number of zeros of the Riemann zeta function in the critical strip in the upper half plane to height $T$. That is, $N(T)$ denotes the number of complex zeros in the rectangle $0 \leq \operatorname{Re} s \leq 1$ and $0 \leq \operatorname{Im} s \leq T$.

Backlund [2] showed that $N(T)$ satisfies

$$
N(T)=\frac{T}{2 \pi} \ln \left(\frac{T}{2 \pi}\right)-\frac{T}{2 \pi}+\frac{7}{8}+e(T),
$$

where

$$
|e(T)|<0.137 \ln T+0.443 \ln \ln T+4.35 \quad \text { for } T \geq 2 .
$$

We believe then that if one were to assume certain statistical properties of the distribution of the Riemann zeros, Conjectures 2 and 3 would follow.

From Eqs. (J.1) and (J.2) we can show that there is a constant $T_{0}$ such that

$$
\pi[N(T+1)-N(T)] \leq \pi \ln T \quad \text { for } T \geq T_{0} .
$$

If we write $N(T)=M(T)+e(T)$, then

$M(T+1)-M(T)=\frac{1}{2 \pi} \ln \left(\frac{T}{2 \pi}\right)+\frac{1}{2 \pi}\left(\frac{1}{2 T}-\frac{1}{6 T^{2}}+\frac{1}{12 T^{3}}-\cdots\right)<\frac{1}{2 \pi} \ln \left(\frac{T}{2 \pi}\right)+\frac{1}{4 \pi T}$.

Then with Eq. (J.2) we have

$N(T+1)-N(T)<\frac{1}{2 \pi} \ln \left(\frac{T}{2 \pi}\right)+\frac{1}{4 \pi T} 0.137\left[2 \ln T+\ln \left(1+\frac{1}{T}\right)\right]+0.866 \ln \ln (T+1)+8.70$, 


$$
T \geq 2 .
$$

Since $\ln (1+1 / T)<1 / T$, we find

$$
N(T+1)-N(T)<0.433 \ln T+0.866 \ln \ln (T+1)+8.407+\frac{0.216}{T} .
$$

We also have that

$$
\begin{gathered}
0.866 \ln \ln (T+1) \leq 0.135 \ln T \quad \text { for } T \geq T_{0}, \\
8.407 \leq 0.431 \ln T \quad \text { for } T \geq T_{0},
\end{gathered}
$$

and we may take $T_{0}=3 \times 10^{8}$. These relations yield inequality (J.3).

We now assume that the Riemann hypothesis holds and consider one possible bound that may result for the sums $\sigma_{k}=\sum_{j} \rho_{j}^{-k}$, where $\left\{\rho_{j}\right\}$ represents the nontrivial zeros of the zeta function. The nontrivial zeros have the form $\rho_{j}=1 / 2+\epsilon+i \alpha_{j}$, where bounds for $\epsilon$ exist in the literature due to results on zero-free regions. As mentioned in the text, a zero $\rho_{j}$ enters the sum $\sigma_{k}$ along with its complex conjugate. We then consider the sums

$$
\sum_{j=m}^{\infty} \frac{1}{\alpha_{j}^{k}} \leq \frac{1}{(k-1)^{2}} \frac{\left[1-\ln \left(\left[\alpha_{m}\right]-1\right)+k \ln \left(\left[\alpha_{m}\right]-1\right)\right]}{\left(\left[\alpha_{m}\right]-1\right)^{k-1}}, \quad\left[\alpha_{m}\right] \geq T_{0}
$$

as an approximation to $\sigma_{k}$, where $[x]$ denotes the greatest integer contained within $x$. We have

$$
\begin{gathered}
\sum_{j=m}^{\infty} \frac{1}{\alpha_{j}^{k}} \leq \sum_{j=\left[\alpha_{m}\right]}^{\infty} \sum_{j \leq \alpha_{\ell}<j+1} \frac{1}{\alpha_{\ell}^{k}} \\
\leq \sum_{j=\left[\alpha_{m}\right]}^{\infty} \frac{[N(j+1)-N(j)]}{j^{k}} \leq \sum_{j=\left[\alpha_{m}\right]}^{\infty} \frac{\ln j}{j^{k}}, \quad \text { for } \quad\left[\alpha_{m}\right] \geq T_{0},
\end{gathered}
$$


where we applied inequality (J.3). Since the last sum in inequality (J.10) is bounded by

$$
\int_{\left[\alpha_{m}\right]-1}^{\infty} \frac{\ln u}{u^{k}} d u=\frac{1}{(k-1)^{2}} \frac{\left[1-\ln \left(\left[\alpha_{m}\right]-1\right)+k \ln \left(\left[\alpha_{m}\right]-1\right)\right]}{\left(\left[\alpha_{m}\right]-1\right)^{k-1}},
$$

we obtain inequality (J.9). 


\section{Appendix K: Observations concerning a Dedekind xi function}

We note here some relations concerning a Dedekind xi function $\xi_{k}$, extending some of our earlier derivative results for the Riemann xi function [9].

We let $k$ be an imaginary quadratic field of discriminant $d$, and

$$
\Phi(t)=\frac{\pi}{\sqrt{|d|}} \sum_{F} \sum_{m, n=-\infty}^{\infty} F(m, n)\left[\frac{\pi t}{\sqrt{|d|}} F(m, n)-1\right] \exp \left[-\frac{2 \pi t}{\sqrt{|d|}} F(m, n)\right]
$$

where the first sum is over the inequivalent classes of positive definite integral quadratic forms of discriminant $d$. Then Li [27] has very recently shown that

$$
\xi_{k}(s)=\frac{4}{w} \int_{1}^{\infty} \Phi(t)\left(t^{s}+t^{1-s}\right) d t
$$

for all complex $s$, where $w$ is the number of roots of unity. The function $\xi_{k}$ is entire and satisfies $\xi_{k}(s)=\xi_{k}(1-s)$ and $\xi_{k}(0)=\xi_{k}(1)=2^{r_{1}} h R / w$, where the number of real places $r_{1}=0$, the number of complex places $r_{2}=1, R=1$ is the regulator, and $h$ is the number of ideal classes of $k$.

The inequivalent classes of positive definite integral quadratic forms of discriminant $d$ consist of the classes represented by the forms [18, 27] $F(x, y)$ satisfying $b^{2}-4 a c=d$ for either $-a<b \leq a<c$ or $0 \leq b \leq a=c$. Now it has also been shown [27] that $F(x, y)=a x^{2}+b x y+c y^{2}$ satisfying this condition is such

that $F(m, n) \geq \sqrt{|d|} / 2$ for all integers $m, n$ with $n \neq 0$. It follows trivially that $\pi t F(m, n) / \sqrt{|d|}-1 \geq \pi t / 2-1>0$ for all $t \in[1, \infty)$. We then have the immediate Proposition. For all the inequivalent classes of positive definite integral quadratic forms of discriminant $d$, we have $\xi_{k}(s)>0$ for all real $s$. Furthermore, the integer- 
order derivatives

$$
\xi_{k}^{(m)}(s)=\frac{4}{w} \int_{1}^{\infty} \Phi(t)\left[t^{s}+(-1)^{m} t^{1-s}\right] \ln ^{m} t d t
$$

satisfy $\xi_{k}^{(m)}(s) \geq 0$ for all $s \geq 1 / 2$. The even order derivatives obey the condition $\xi_{k}^{(2 m)}(s)>0$ for all $s \geq 1 / 2$. Of course, as also seen by the functional equation for $\xi_{k}(s)$, the odd-order derivatives $\xi_{k}^{(2 m+1)}$ vanish at $s=1 / 2$; we have $\xi_{k}^{(m)}(s)=$ $(-1)^{m} \xi_{k}^{(m)}(1-s)$. This Proposition seems to mean that $\xi_{k}(s)$ has no zeros for real values of $s$.

This Proposition extends some of the results of Ref. [9]. In turn, we may apply some of the explicit integration results obtained there in order to evaluate the function

$$
\gamma(a) \equiv \int_{1}^{\infty} \omega(t) \frac{d t}{\sqrt{t}}+\int_{1}^{\sqrt{|d|} / 2 a} \omega(t) \frac{d t}{t}
$$

introduced in Ref. [27], where $\omega$ is the $\theta$ series given by $\omega(t)=\sum_{n=1}^{\infty} \exp \left(-\pi n^{2} t\right)$. We have for the first term on the right side of Eq. (K.4)

$$
\sum_{n=1}^{\infty} \int_{1}^{\infty} e^{-\pi n^{2} t} t^{-1 / 2} d t=\frac{1}{\sqrt{\pi}} \sum_{n=1}^{\infty} \frac{1}{n} \Gamma\left(1 / 2, n^{2} \pi\right)=\sum_{n=1}^{\infty} \frac{1}{n}[1-\operatorname{Erf}(n \sqrt{\pi})]
$$

where $\Gamma(x, y)$ is the incomplete Gamma function [16] and $\Gamma\left(1 / 2, n^{2} \pi\right)=\sqrt{\pi}[1-$ $\left.2 n{ }_{1} F_{1}\left(1 / 2 ; 3 / 2 ;-\pi n^{2}\right)\right]$ and ${ }_{1} F_{1}$ is the confluent hypergeometric function $[16,3]$, such that ${ }_{1} F_{1}(1 / 2 ; 3 / 2 ; x)=(\sqrt{\pi} / 2) \operatorname{Er} f(\sqrt{-x}) / \sqrt{-x}$, where $\operatorname{Er} f$ is the error function (probability integral) [16].

For the second term on the right side of Eq. (K.4) we have

$$
\sum_{n=1}^{\infty} \int_{1}^{\sqrt{|d|} / 2 a} e^{-\pi n^{2} t} t^{-1} d t=\sum_{n=1}^{\infty}\left[E i\left(-\frac{\pi n^{2} \sqrt{|d|}}{2 a}\right)-E i\left(-\pi n^{2}\right)\right]
$$


where $E i$ is the exponential integral [16]. We also have various elementary relations, including

$$
\int_{1}^{\sqrt{|d|} / 2 a} e^{-\pi n^{2} t} t^{-1} d t=\pi n^{2} \int_{1}^{\sqrt{|d|} / 2 a} e^{-\pi n^{2} t} \ln t d t+\exp \left(-\pi n^{2} \sqrt{|d|} / 2 a\right) \ln \left(\frac{\sqrt{|d|}}{2 a}\right)
$$

obtained by integration by parts, and

$$
\begin{gathered}
\int_{1}^{\sqrt{|d|} / 2 a} e^{-\pi n^{2} t} \ln t d t=\frac{1}{\pi n^{2}} \int_{\pi n^{2}}^{\pi n^{2} \sqrt{|d|} / 2 a} e^{-u}\left[\ln u-\ln \left(\pi n^{2}\right)\right] d t \\
=\frac{1}{\pi n^{2}}\left[\int_{\pi n^{2}}^{\pi n^{2} \sqrt{|d|} / 2 a} e^{-u} \ln u d u-\left(\exp \left(-\pi n^{2} \sqrt{|d|} / 2 a\right)-\exp \left(-\pi n^{2}\right)\right) \ln \left(\pi n^{2}\right)\right] .
\end{gathered}
$$

Moreover, the particular derivative values

$$
\xi_{k}^{(2 m)}\left(\frac{1}{2}\right)=\frac{8}{w} \int_{1}^{\infty} \Phi(t) t^{1 / 2} \ln ^{2 m} t d t
$$

and

$$
\xi_{k}^{(m)}(1)=\frac{4}{w} \int_{1}^{\infty} \Phi(t)\left[t+(-1)^{m}\right] \ln ^{m} t d t
$$

can be evaluated in terms of infinite series with the analytic methods of Ref. [9], and we note that $\xi_{k}^{(m)}(1)>0$ for all nonnegative integers $m$. These special values of Eq. (K.10) enter the particular logarithmic derivatives of Eq. (E.31) for $\lambda_{n}$. 


\section{Appendix L: Euler-Maclaurin summation applied to $S_{1}(n)$}

Here we apply Euler-Maclaurin summation to the form of the sum $S_{1}(n)$ given in Eq. (17) of the text. Accordingly, we define the summand function

$$
f(k) \equiv \frac{n}{2 k+1}-1+\frac{2^{n} k^{n}}{(2 k+1)^{n}}, \quad k \geq 0, \quad n \geq 2
$$

such that $f(0)=n-1$ and $f(\infty)=0$. We can write the arbitrary integer order derivative of each term of Eq. (L.1). For the first term on the right side we have

$$
\left(\frac{d}{d k}\right)^{j} \frac{n}{(2 k+1)}=(-1)^{j} \frac{2^{j} j !}{(2 k+1)^{j+1}} n, \quad j \geq 1 .
$$

When evaluated at $k=0$, this term gives $(-1)^{j} 2^{j} j ! n$. In regard to the last term of Eq. (L.1) we have

$$
\left(\frac{d}{d k}\right)^{j} \frac{1}{(2 k+1)^{n}}=(-1)^{j} \frac{2^{j}(n)_{j}}{(2 k+1)^{n+j}}, \quad j \geq 1
$$

where $(.)_{j}$ is the Pochhammer symbol, and

$$
\left(\frac{d}{d k}\right)^{r} k^{n}=\frac{n !}{(n-r) !} k^{n-r}
$$

which can be equally expressed as

$$
\left(\frac{d}{d k}\right)^{r} k^{n}=r !\left(\begin{array}{l}
n \\
r
\end{array}\right) k^{n-r}=(-1)^{r}(-n)_{r} k^{n-r} .
$$

Therefore we can write

$$
2^{n}\left(\frac{d}{d k}\right)^{\ell} k^{n}(2 k+1)^{-n}=2^{n} \sum_{m=n}^{\ell}\left(\begin{array}{c}
\ell \\
m
\end{array}\right) \frac{(-1)^{\ell-m} 2^{\ell-m}(n)_{\ell-m}}{(2 k+1)^{n+\ell-m}} \frac{n !}{(n-m) !} k^{n-m} .
$$


A version of the Euler-Maclaurin formula, given that all derivatives of $f$ vanish at infinity, is

$$
\sum_{n=M}^{\infty} f(n)=\int_{M}^{\infty} f(x) d x-\sum_{m=1}^{\infty} \frac{B_{m}}{m !} f^{(m-1)}(M)
$$

where $B_{m}$ are Bernoulli numbers, or

$$
\sum_{n=0}^{\infty} f(n)=\int_{0}^{\infty} f(x) d x+\frac{1}{2} f(0)-\sum_{m=2, \text { even }}^{\infty} \frac{B_{m}}{m !} f^{(m-1)}(0) .
$$

By using Eq. (24) of the text for the integral in Eq. (L.5b) we therefore obtain

$$
S_{1}(n)=\frac{n}{2}[\psi(n)+\gamma]-\sum_{m=2, \text { even }}^{\infty} \frac{B_{m}}{m !} f^{(m-1)}(0),
$$

where $\psi$ is the digamma function and $\gamma$ is the Euler constant. The sums in Eqs. (L.5)(L.6) are meant in an asymptotic sense; they are highly unlikely to be convergent. From Eq. (L.6) we may obtain the successive approximations

$$
S_{1}(n)=\frac{n}{2}[\psi(n)+\gamma]+\frac{n}{6}-\sum_{m=4, \text { even }}^{\infty} \frac{B_{m}}{m !} f^{(m-1)}(0), \quad n>1,
$$

and

$$
S_{1}(n)=\frac{n}{2}[\psi(n)+\gamma]+\frac{n}{10}-\sum_{m=6, \text { even }}^{\infty} \frac{B_{m}}{m !} f^{(m-1)}(0), \quad n>3 .
$$

Equation (L.7a) is expected to be a useful approximate upper bound to $S_{1}$ and Eq. (L.7b) an approximate lower bound for this sum. When $n$ is sufficiently large, only the first term on the right side of Eq. (L.1) contributes in Eqs. (L.6)-(L.7), giving $B_{m} f^{(m-1)}(0) / m !=-2^{m-1} B_{m}(n / m)$, as $m-1$ is always an odd integer. Given alternation in sign from $B_{2 m}$ to $B_{2 m+2}$, these successive terms will change sign also. However, we emphasize that the sums in Eq. (L.6)-(L.7) are generally divergent. 


\section{References}

[1] M. Abramowitz and I. A. Stegun, Handbook of Mathematical Functions, Washington, National Bureau of Standards (1964).

[2] R. J. Backlund, Über die Nullstellen der Riemannschen Zetafunktion, Acta Math. 41, 345-375 (1918).

[3] W. N. Bailey, Generalized Hypergeometric Series, Cambridge University Press (1935); G. E. Andrews, R. Askey, and R. Roy, Special Functions, Cambridge University Press (1999).

[4] B. C. Berndt, On the Hurwitz zeta function, Rocky Mtn. J. Math. 2, 151-157 (1972).

[5] P. Biane, J. Pitman, and M. Yor, Probability laws related to the Jacobi theta and Riemann zeta functions and Brownian excursions, Bull. Amer. Math. Soc. 38, 435-465 (2001).

[6] E. Bombieri and J. C. Lagarias, Complements to Li's criterion for the Riemann hypothesis, J. Number Theory 77, 274-287 (1999).

[7] D. Bump, Automorphic Forms and Representations, Cambridge University Press (1997).

[8] W. E. Clark and M. E. H. Ismail, Inequalities involving gamma and psi functions, http://www.math.usf.edu/ clark, to appear in Analysis and Applications. 
[9] M. W. Coffey, Relations and positivity results for derivatives of the Riemann $\xi$ function, J. Comput. Appl. Math., 166, 525-534 (2004).

[10] M. W. Coffey, On some log-cosine integals related to $\zeta(2), \zeta(3)$, and $\zeta(6)$, J. Comput. Appl. Math. 159, 205-215 (2003).

[11] M. W. Coffey, New results on the Li constants, preprint (2004)

[12] H. Davenport, Multiplicative Number Theory, Springer Verlag (2000).

[13] H. M. Edwards, Riemann's Zeta Function, Academic Press, New York (1974).

[14] E. Elizalde, An asymptotic expansion for the first derivative of the generalized Riemann zeta function, Math. Comp. 47, 347-350 (1986).

[15] P. Flajolet and R. Sedgewick, Mellin transforms and asymptotics: Finite differences and Rice's integrals, Th. Comp. Sci. 144, 101-124 (1995).

[16] I. S. Gradshteyn and I. M. Ryzhik, Table of Integrals, Series, and Products, Academic Press, New York (1980).

[17] R. L. Graham, D. E. Knuth, and O. Patashnik, Concrete Mathematics, 2nd ed., Addison Wesley (1994).

[18] L. K. Hua, Introduction to Number Theory, Springer Verlag (1982).

[19] M. I. Israilov, On the Laurent Decomposition of Riemann's zeta function, Dokl. Akad. Nauk SSSR (Russian) 12, 9 (1979); M. I. Israilov, Trudy Mat. Inst. 
Steklova 158, 98-104 (1981); G. H. Hardy, Note on Dr. Vacca's series for $\gamma$, Quart. J. Pure Appl. Math. 43, 215-216 (1912); J. C. Kluyver, On certain series of Mr. Hardy, Quart. J. Pure Appl. Math. 50, 185-192 (1927); W. E. Briggs, Some constants associated with the Riemann zeta-function, Mich. Math. J. 3, 117-121 (1955); D. Mitrović, The signs of some constants associated with the Riemann zeta function, Mich. Math. J. 9, 395-397 (1962).

[20] A. Ivić, The Riemann Zeta-Function, Wiley (1985).

[21] A. Ivić, The Laurent coefficients of certain Dirichlet series, Publs. Inst. Math. 53, 23-36 (1993). In Eq. (1.7), $(\log x)^{x}$ should be replaced with $(\log x)^{k}$ and on p. 25 "comparing with (1.15) should be replaced with "comparing with (1.5)".

[22] A. A. Karatsuba and S. M. Voronin, The Riemann Zeta-Function, Walter de Gruyter, New York (1992).

[23] D. E. Knuth, The Art of Computer Programming, Vol. 3, Addison Wesley (1973).

[24] D. H. Lehmer, The sum of like powers of the zeros of the Riemann zeta function, Math. Comput. 50, 265-273 (1988).

[25] X.-J. Li, The positivity of a sequence of numbers and the Riemann hypothesis, J. Number Th. 65, 325-333 (1997).

[26] X.-J. Li, Explicit formulas for Dirichlet and Hecke L-functions, to appear in Ill. J. Math. (2004). 
[27] X.-J. Li, A formula for the Dedekind $\xi$-function of an imaginary quadratic field, J. Math. Anal. Appl. 260, 404-420 (2001).

[28] Y. L. Luke, The Special Functions and Their Approximations, Academic Press (1969).

[29] K. Maślanka, http://nac.oa.uj.edu.pl/masaslanka; Effective method of computing Li's coefficients and their properties, to appear in Exp. Math. (2004).

[30] J.-F. Mestre, Formules explicites et minorations de conducteurs de variétés algébriques, Compositio Math. 58, 209-232 (1986).

[31] Z. Nan-Yue and K. S. Williams, Some results on the generalized Stieltjes constants, Analysis 14, 147-162 (1994). In addition to the typographical errors pointed out in Appendix C, the first term on the right side of Eq. (6.2) should read $\left(\log ^{n} a\right) / a$, and in both Eqs. (6.5) and (6.11), $P_{n}$ should appear in place of $P_{1}$. On p. 157 of this reference, (7.2) should be replaced with (7.1) in the second line of text from the bottom, and on p. 158 (7.2) should be replaced with (1.9) in the third line of text.

[32] J. Neukirch, Algebraic Number Theory, Springer Verlag (1999).

[33] A. M. Odlyzko, http://www.dtc.umn.edu/õdlyzko/ 
[34] A. M. Odlyzko, Bounds for discriminants and related estimates for class numbers, regulators and zeros of zeta functions: A survey of recent results, Sem. Th. No., Bordeaux, g, 1-15 (1989).

[35] S. Omar, Localization of the first zero of the Dedekind zeta function, Math. Comp. 70, 1607-1616 (2001).

[36] K. S. Rao, G. V. Berghe, and C. Krattenthaler, An entry of Ramanujan on hypergeometric series in his notebooks, preprint arXiv:math.CA/0304317 v1 (2003).

[37] B. Riemann, Über die Anzahl der Primzahlen unter einer gegebenen Grösse, Monats. Preuss. Akad. Wiss., 671 (1859-1860).

[38] E. C. Titchmarsh, The Theory of the Riemann Zeta-Function, 2nd ed., Oxford University Press, Oxford (1986).

[39] A. Voros, A sharpening of Li's criterion for the Riemann hypothesis, arXiv:math.NT/0404213 (2004)

[40] A. Weil, Basic Number Theory, Springer Verlag (1967). 
This figure "lambda2fig1.jpg" is available in "jpg" format from: http://arxiv.org/ps/math-ph/0505052v1 